\title{
Optimization of Hybrid Laminates with Extension-Shear Coupling
}

\author{
Da Cui $(\mathbb{D})$ and Daokui Li $(\mathbb{D}$ \\ College of Aerospace Science and Engineering, National University of Defense Technology, Changsha 410073, China \\ Correspondence should be addressed to Daokui Li; lidaokui@nudt.edu.cn
}

Received 25 February 2018; Revised 11 April 2018; Accepted 17 April 2018; Published 27 May 2018

Academic Editor: Roberto G. Citarella

Copyright (c) 2018 Da Cui and Daokui Li. This is an open access article distributed under the Creative Commons Attribution License, which permits unrestricted use, distribution, and reproduction in any medium, provided the original work is properly cited.

\begin{abstract}
The introduction of hybrid composites into the structure with coupling effect can greatly reduce the cost of materials. The expressions of stiffness coefficient, thermal stress, and thermal moment for hybrid laminates are derived based on the geometrical factors of laminates, and the necessary and sufficient conditions for the hybrid extension-shear-coupled laminates with immunity to hygrothermal shear distortion (HTSD) are further derived. The extension-shear-coupled effect of hybrid laminates is optimized with improved differential evolution algorithm. Results are presented for the hybrid laminates that consist of carbon fiber and glass fiber composite materials. The hygrothermal effect and extension-shear-coupled effect are simulated and verified, meanwhile the robustness of hybrid laminates is analyzed by Monte Carlo method.
\end{abstract}

\section{Introduction}

Laminated composites are playing an important and irreplaceable role in designing structures with coupling effect. For example, the bending-twisting-coupled wing structure can be designed by using composite extension-shearcoupled laminates [1] and the bending-twisting-coupled wind turbine blades structure can be designed by using composite extension-twisting-coupled laminates [2]. However, with the large-scale use of these bending-twisting coupling structures, the common glass fiber composites have difficulties in meeting the requirements of structural reliability. On the other hand, the carbon fiber composites with good comprehensive properties $[3,4]$ cost about ten times more than glass fiber composites, which restricts its wide range of applications. Therefore, it is necessary to introduce hybrid fiber composites into the design of the bendingtwisting-coupled structure to achieve the purpose that greatly reduces material cost under meeting the structural reliability requirements.

According to different hybrid modes, hybrid fiber composites are mainly divided into two types of composites, the in-layer composites and between-layer composites [5]. The in-layer hybrid composites consist of two or more fibers which uniformly dispersed in the same matrix of the lamina; the between-layer hybrid composites are composed of two or more different laminae which consist of different single-fiber composites. In this paper, the bending-twisting-coupled structure is designed by between-layer hybrid laminates.

At present, the widely used materials in the study of the bending-twisting coupled structure are the single-fiber composite laminates [6-10], whose important design parameters contain paving angles merely. However, the important design parameters for hybrid laminates also include the paving materials [11]. Once the paving materials become variable, the design of laminates becomes more complicated and the number of optimization constraints even multiplies at the same time.

J. Li and D. Li [2] have designed a kind of single-fiber composite laminates with immunity to hygrothermal shear distortion (HTSD) with only extension-shear-coupled effect-the $\mathbf{A}_{F} \mathbf{B}_{0} \mathbf{D}_{S}$ laminates (refer to the nomenclature of references $[1,2]$ ) - and the sequential quadratic programming (SQP) is used to optimize its coupled effect. However, we found that feasible solutions cannot be found when using the SQP algorithm to optimize the hybrid $\mathbf{A}_{F} \mathbf{B}_{0} \mathbf{D}_{S}$ laminates with immunity to HTSD, for this algorithm cannot meet the strong constraints of hybrid laminates. Therefore, this paper 
takes the improved differential evolution algorithm DE_CMSBHS to optimize this problem.

The DE_CMSBHS algorithm is an efficient global optimization algorithm, which has the characteristics of simple structure, easy realization, fast convergence, and strong robustness, and can effectively solve the single-objective optimization problems such as integer problems, real problems, and mixed integer-real problems [12]. The single-objective nonlinear optimization problems are able to be solved well by combining the penalty function's ability to handle constraints with the optimal performance of DE_CMSBHS algorithm $[13,14]$. The optimization problem of hybrid $\mathbf{A}_{F} \mathbf{B}_{0} \mathbf{D}_{S}$ laminates with immunity to HTSD is a typical nonlinear mixed integer-real single-objective optimization problem.

In this paper, the model of hybrid laminates is firstly established. Then the expressions of stiffness coefficient, thermal stress, and thermal moment for laminates are derived with important parameter geometric factor $[15,16]$. Secondly, the necessary and sufficient conditions for the hybrid extension-shear-coupled laminates with immunity to HTSD are derived. Thirdly, the DE_CMSBHS algorithm combined with the penalty function is used to optimize the extensionshear-coupled effect of hybrid laminates with immunity to HTSD. Finally, the mechanical properties of optimized laminates are verified.

\section{Stiffness Coefficient, Thermal Stress, and Thermal Moment of Hybrid Laminates}

Introducing the geometrical factors into the design of laminates can effectively improve the efficiency of this progress. In this section, the model of hybrid laminates will be established. Based on the geometric factors, the expressions of stiffness coefficient, thermal stress, and thermal moment of hybrid laminates are derived.

The research object is set as a kind of hybrid laminates which are composed of two different types of lamina, and each lamina has the same thickness, as shown in Figure 1. Wherein, $z_{k}$ is the position of the $k$-ply in the entire laminates, $n$ is the number of plies of the laminates, and $H$ is the entire thickness of the laminates. (1) and (2) represent two kinds of laminae with different material properties, respectively, and the number and layer order of each kind of lamina are variables.

2.1. Stiffness Coefficient. The off-axis stiffness coefficients of the $k$-ply in the entire hybrid laminates can be defined as

$$
\begin{aligned}
& \left(\bar{Q}_{11}\right)_{k}=U_{1}^{q}+U_{2}^{q} \cos 2 \theta_{k}+U_{3}^{q} \cos 4 \theta_{k}, \\
& \left(\bar{Q}_{12}\right)_{k}=-U_{3}^{q} \cos 4 \theta_{k}+U_{4}^{q}, \\
& \left(\bar{Q}_{16}\right)_{k}=\frac{U_{2}^{q}}{2} \sin 2 \theta_{k}+U_{3}^{q} \sin 4 \theta_{k}, \\
& \left(\bar{Q}_{22}\right)_{k}=U_{1}^{q}-U_{2}^{q} \cos 2 \theta_{k}+U_{3}^{q} \cos 4 \theta_{k}, \\
& \left(\bar{Q}_{26}\right)_{k}=\frac{U_{2}^{q}}{2} \sin 2 \theta_{k}-U_{3}^{q} \sin 4 \theta_{k}, \\
& \left(\bar{Q}_{66}\right)_{k}=-U_{3}^{q} \cos 4 \theta_{k}+U_{5}^{q} .
\end{aligned}
$$

Wherein, $\theta_{k}$ is the paving angle of the $k$-ply of the laminates. The superscript " $q$ " is defined to mean which kind of lamina corresponds to: $q=$ (1) implies that the lamina corresponds to the type (1) lamina and $q=$ (2) implies that the lamina corresponds to the type (2) lamina. Moreover, $U_{i}^{q}(i=1,2, \ldots, 5)$ are the material constants of hybrid laminates, which are only related to the material parameters of the laminae, as shown in

$$
\begin{aligned}
U_{1}^{q} & =\frac{\left(3 Q_{11}^{q}+3 Q_{22}^{q}+2 Q_{12}^{q}+4 Q_{66}^{q}\right)}{8}, \\
U_{2}^{q} & =\frac{\left(Q_{11}^{q}-Q_{22}^{q}\right)}{2}, \\
U_{3}^{q} & =\frac{\left(Q_{11}^{q}+Q_{22}^{q}-2 Q_{12}^{q}-4 Q_{66}^{q}\right)}{8}, \\
U_{4}^{q} & =\frac{\left(Q_{11}^{q}+Q_{22}^{q}+6 Q_{12}^{q}-4 Q_{66}^{q}\right)}{8}, \\
U_{5}^{q} & =\frac{\left(Q_{11}^{q}+Q_{22}^{q}-2 Q_{12}^{q}+4 Q_{66}^{q}\right)}{8} \\
& =\frac{1}{2}\left(U_{1}^{q}-U_{4}^{q}\right) .
\end{aligned}
$$

In which, $Q_{i j}^{q}$ are the stiffness coefficients of two types of lamina. The geometric factors $\xi_{j}^{q}(j=1,2, \ldots, 15, q=(1)$, (2) of hybrid laminates, which are convenient to express the stiffness matrices, are defined as

$$
\begin{aligned}
& \left(\xi_{1}^{q} \xi_{2}^{q} \xi_{3}^{q} \xi_{4}^{q}\right)=\sum_{k=q}\left(\begin{array}{llll}
\cos 2 \theta_{k} & \cos 4 \theta_{k} & \sin 2 \theta_{k} & \sin 4 \theta_{k}
\end{array}\right) \\
& \left(z_{k}-z_{k-1}\right) \\
& \left(\begin{array}{llll}
\xi_{5}^{q} & \xi_{6}^{q} & \xi_{7}^{q} & \xi_{8}^{q}
\end{array}\right)=\sum_{k=q}\left(\begin{array}{llll}
\cos 2 \theta_{k} & \cos 4 \theta_{k} & \sin 2 \theta_{k} & \sin 4 \theta_{k}
\end{array}\right) \\
& \left(z_{k}^{2}-z_{k-1}^{2}\right) \\
& \left(\begin{array}{llll}
\xi_{9}^{q} & \xi_{10}^{q} & \xi_{11}^{q} & \xi_{12}^{q}
\end{array}\right)=\sum_{k=q}\left(\begin{array}{llll}
\cos 2 \theta_{k} & \cos 4 \theta_{k} & \sin 2 \theta_{k} & \sin 4 \theta_{k}
\end{array}\right) \\
& \left(z_{k}^{3}-z_{k-1}^{3}\right) \\
& \left(\begin{array}{lll}
\xi_{13}^{q} & \xi_{14}^{q} & \xi_{15}^{q}
\end{array}\right)=\sum_{k=q}\left[\begin{array}{lll}
\left(z_{k}-z_{k-1}\right) & \left(z_{k}^{2}-z_{k-1}^{2}\right) & \left(z_{k}^{3}-z_{k-1}^{3}\right)
\end{array}\right],
\end{aligned}
$$

where $k=q$ means the sum of all the type (1) or (2) laminae on the right side of the equation. Obviously, the geometric factors are only related to the paving angle and location of each lamina.

2.1.1. Extension Stiffness Matrix A. According to the classical theory of laminates, the extension stiffness matrix of laminates can be expressed as

$$
\mathbf{A}_{i j}=\sum_{k=1}^{n}\left(\bar{Q}_{i j}\right)_{k}\left(z_{k}-z_{k-1}\right), \quad i, j=1,2,6
$$

Substituting (1), (2), and (3) into (4) can be used to obtain the expressions of the extension stiffness coefficients, which 


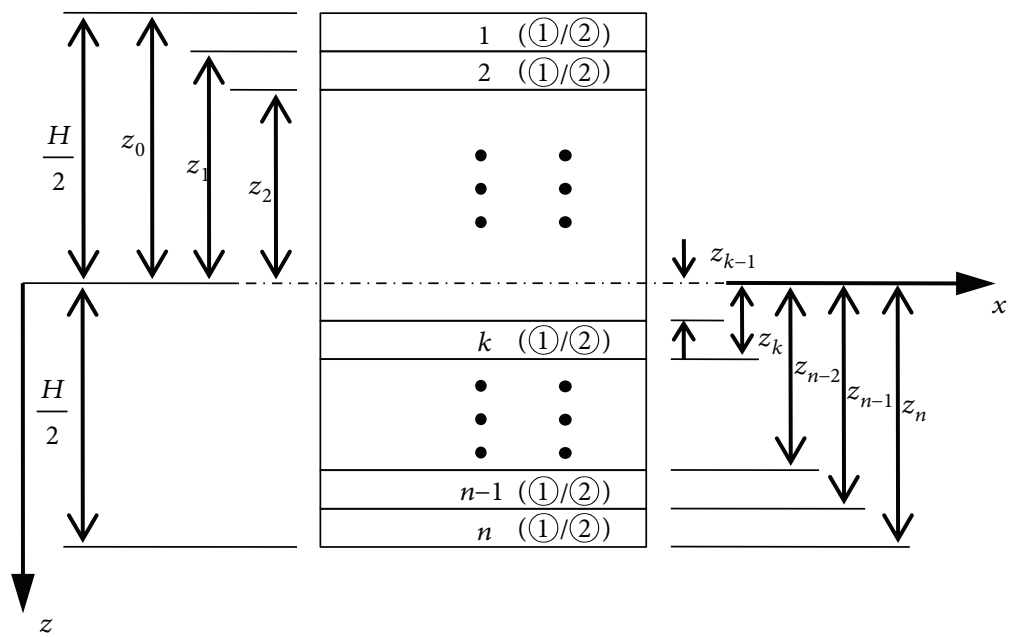

Figure 1: Model of hybrid laminates.

are only related to the material constants and geometric factors, as shown in

$$
\begin{aligned}
& \mathbf{A}_{11}=\sum_{k=1}^{n}\left(U_{1}^{q}+U_{2}^{q} \cos 2 \theta_{k}+U_{3}^{q} \cos 4 \theta_{k}\right)\left(z_{k}-z_{k-1}\right) \\
& =U_{1}^{(1)} \xi_{13}^{(1)}+U_{2}^{(1)} \xi_{1}^{(1)}+U_{3}^{(1)} \xi_{2}^{(1)}+U_{1}^{(2)} \xi_{13}^{(2)}+U_{2}^{(2)} \xi_{1}^{(2)}+U_{3}^{(2)} \xi_{2}^{(2)} \text {, } \\
& \mathbf{A}_{12}=\mathbf{A}_{21}=\sum_{k=1}^{n}\left[\left(-\cos 4 \theta_{k}\right) U_{3}^{q}+U_{4}^{q}\right]\left(z_{k}-z_{k-1}\right) \\
& =-U_{3}^{(1)} \xi_{2}^{(1)}+U_{4}^{(1)} \xi_{13}^{(1)}-U_{3}^{(2)} \xi_{2}^{(2)}+U_{4}^{(2)} \xi_{13}^{(2)} \\
& \mathbf{A}_{16}=\mathbf{A}_{61}=\sum_{k=1}^{n}\left[U_{2}^{q}\left(\frac{\sin 2 \theta_{k}}{2}\right)+U_{3}^{q} \sin 4 \theta_{k}\right]\left(z_{k}-z_{k-1}\right) \\
& =\frac{1}{2} U_{2}^{(1)} \xi_{3}^{(1)}+U_{3}^{(1)} \xi_{4}^{(1)}+\frac{1}{2} U_{2}^{(2)} \xi_{3}^{(2)}+U_{3}^{(2)} \xi_{4}^{(2)} \text {, } \\
& \mathbf{A}_{22}=\sum_{k=1}^{n}\left(U_{1}^{q}-U_{2}^{q} \cos 2 \theta_{k}+U_{3}^{q} \cos 4 \theta_{k}\right)\left(z_{k}-z_{k-1}\right) \\
& =U_{1}^{(1)} \xi_{13}^{(1)}-U_{2}^{(1)} \xi_{1}^{(1)}+U_{3}^{(1)} \xi_{2}^{(1)}+U_{1}^{(2)} \xi_{13}^{(2)}-U_{2}^{(2} \xi_{1}^{(2)}+U_{3}^{(2} \xi_{2}^{(2}, \\
& \mathbf{A}_{26}=\mathbf{A}_{62}=\sum_{k=1}^{n}\left[U_{2}^{q}\left(\frac{\sin 2 \theta_{k}}{2}\right)-U_{3}^{q} \sin 4 \theta_{k}\right]\left(z_{k}-z_{k-1}\right) \\
& =\frac{1}{2} U_{2}^{(1)} \xi_{3}^{(1)}-U_{3}^{(1)} \xi_{4}^{(1)}+\frac{1}{2} U_{2}^{(2)} \xi_{3}^{(2)}-U_{3}^{(2)} \xi_{4}^{(2)} \text {, } \\
& \mathbf{A}_{66}=\sum_{k=1}^{n}\left(-U_{3}^{q} \cos 4 \theta_{k}+U_{5}^{q}\right)\left(z_{k}-z_{k-1}\right) \\
& =-U_{3}^{(1)} \xi_{2}^{(1)}+U_{5}^{(1)} \xi_{13}^{(1)}-U_{3}^{(2)} \xi_{2}^{(2)}+U_{5}^{(2)} \xi_{13}^{(2)}
\end{aligned}
$$

2.1.2. Coupling Stiffness Matrix B. Similarly, the coupling stiffness matrix of the hybrid laminates can be expressed as

$$
\mathbf{B}_{i j}=\sum_{k=1}^{n}\left(\bar{Q}_{i j}\right)_{k}\left(z_{k}^{2}-z_{k-1}^{2}\right), \quad i, j=1,2,6 .
$$

Substituting (1), (2), and (3) into (6) can be used to obtain the expression of the coupling stiffness coefficients, which are only related to the material constants and geometric factors, as shown in

$$
\begin{aligned}
\mathbf{B}_{11} & =\frac{1}{2} \sum_{k=1}^{n}\left(U_{1}^{q}+U_{2}^{q} \cos 2 \theta_{k}+U_{3}^{q} \cos 4 \theta_{k}\right)\left(z_{k}^{2}-z_{k-1}^{2}\right) \\
& =\frac{1}{2} U_{1}^{(1)} \xi_{14}^{(1)}+\frac{1}{2} U_{2}^{(1)} \xi_{5}^{(1)}+\frac{1}{2} U_{3}^{(1)} \xi_{6}^{(1)}+\frac{1}{2} U_{1}^{(2)} \xi_{14}^{(2)} \\
& +\frac{1}{2} U_{2}^{(2)} \xi_{5}^{(2)}+\frac{1}{2} U_{3}^{(2)} \xi_{6}^{(2)}, \\
\mathbf{B}_{12} & =\mathbf{B}_{21}=\frac{1}{2} \sum_{k=1}^{n}\left[\left(-\cos 4 \theta_{k}\right) U_{3}^{q}+U_{4}^{q}\right]\left(z_{k}^{2}-z_{k-1}^{2}\right) \\
& =-\frac{1}{2} U_{3}^{(1)} \xi_{6}^{(1)}+\frac{1}{2} U_{4}^{(1)} \xi_{14}^{(1)}-\frac{1}{2} U_{3}^{(2)} \xi_{6}^{(2)}+\frac{1}{2} U_{4}^{(2)} \xi_{14}^{(2)},
\end{aligned}
$$

$$
\begin{aligned}
\mathbf{B}_{22} & =\frac{1}{2} \sum_{k=1}^{n}\left(U_{1}^{q}-U_{2}^{q} \cos 2 \theta_{k}+U_{3}^{q} \cos 4 \theta_{k}\right)\left(z_{k}^{2}-z_{k-1}^{2}\right) \\
& =\frac{1}{2} U_{1}^{(1)} \xi_{14}^{(1)}-\frac{1}{2} U_{2}^{(1)} \xi_{5}^{(1)}+\frac{1}{2} U_{3}^{(1)} \xi_{6}^{(1)}+\frac{1}{2} U_{1}^{(2)} \xi_{14}^{(2)}-\frac{1}{2} U_{2}^{(2)} \xi_{5}^{(2)} \\
& +\frac{1}{2} U_{3}^{(2)} \xi_{6}^{(2)},
\end{aligned}
$$

$$
\begin{aligned}
\mathbf{B}_{16} & =\mathbf{B}_{61}=\frac{1}{2} \sum_{k=1}^{n}\left[U_{2}^{q}\left(\frac{\sin 2 \theta_{k}}{2}\right)+U_{3}^{q} \sin 4 \theta_{k}\right]\left(z_{k}^{2}-z_{k-1}^{2}\right) \\
& =\frac{1}{4} U_{2}^{(1)} \xi_{7}^{(1)}+\frac{1}{2} U_{3}^{(1)} \xi_{8}^{(1)}+\frac{1}{4} U_{2}^{(2)} \xi_{7}^{(2)}+\frac{1}{2} U_{3}^{(2)} \xi_{8}^{(2)}, \\
\mathbf{B}_{26} & =\mathbf{B}_{62}=\frac{1}{2} \sum_{k=1}^{n}\left[U_{2}^{q}\left(\frac{\sin 2 \theta_{k}}{2}\right)-U_{3}^{q} \sin 4 \theta_{k}\right]\left(z_{k}^{2}-z_{k-1}^{2}\right) \\
& =\frac{1}{4} U_{2}^{(1)} \xi_{7}^{(1)}-\frac{1}{2} U_{3}^{(1)} \xi_{8}^{(1)}+\frac{1}{4} U_{2}^{(2)} \xi_{7}^{(2)}-\frac{1}{2} U_{3}^{(2)} \xi_{8}^{(2)},
\end{aligned}
$$

$\mathbf{B}_{66}=\frac{1}{2} \sum_{k=1}^{n}\left(-U_{3}^{q} \cos 4 \theta_{k}+U_{5}^{q}\right)\left(z_{k}^{2}-z_{k-1}^{2}\right)$

$$
=-\frac{1}{2} U_{3}^{(1)} \xi_{6}^{(1)}+\frac{1}{2} U_{5}^{(1)} \xi_{14}^{(1)}-\frac{1}{2} U_{3}^{(2)} \xi_{6}^{(2)}+\frac{1}{2} U_{5}^{(2)} \xi_{14}^{(2)} \text {. }
$$


2.1.3. Bending Stiffness Matrix D. The bending stiffness matrix of the hybrid laminates can be expressed as

$$
\mathbf{D}_{i j}=\sum_{k=1}^{n}\left(\bar{Q}_{i j}\right)_{k}\left(z_{k}^{3}-z_{k-1}^{3}\right), \quad i, j=1,2,6 .
$$

Substituting (1), (2), and (3) into (8) can be used to obtain the expressions of the bending stiffness coefficients, which are only related to the material constants and geometric factors, as shown in

$$
\begin{aligned}
& \mathbf{D}_{11}=\frac{1}{3} \sum_{k=1}^{n}\left(U_{1}^{q}+U_{2}^{q} \cos 2 \theta_{k}+U_{3}^{q} \cos 4 \theta_{k}\right)\left(z_{k}^{3}-z_{k-1}^{3}\right) \\
& =\frac{1}{3} U_{1}^{(1)} \xi_{15}^{(1)}+\frac{1}{3} U_{2}^{(1)} \xi_{9}^{(1)}+\frac{1}{3} U_{3}^{(1)} \xi_{10}^{(1)}+\frac{1}{3} U_{1}^{(2)} \xi_{15}^{(2)}+\frac{1}{3} U_{2}^{(2)} \xi_{9}^{(2)} \\
& +\frac{1}{3} U_{3}^{2} \xi_{10}^{2} \\
& \mathbf{D}_{12}=\mathbf{D}_{21}=\frac{1}{3} \sum_{k=1}^{n}\left[\left(-\cos 4 \theta_{k}\right) U_{3}^{q}+U_{4}^{q}\right]\left(z_{k}^{3}-z_{k-1}^{3}\right) \\
& =-\frac{1}{3} U_{3}^{(1)} \xi_{10}^{(1)}+\frac{1}{3} U_{4}^{(1)} \xi_{15}^{(1)}-\frac{1}{3} U_{3}^{(2)} \xi_{10}^{(2)}+\frac{1}{3} U_{4}^{(2)} \xi_{15}^{(2)} \text {, } \\
& \mathbf{D}_{16}=\mathbf{D}_{61}=\frac{1}{3} \sum_{k=1}^{n}\left[U_{2}^{q}\left(\frac{\sin 2 \theta_{k}}{2}\right)+U_{3}^{q} \sin 4 \theta_{k}\right]\left(z_{k}^{3}-z_{k-1}^{3}\right) \\
& =\frac{1}{6} U_{2}^{(1)} \xi_{11}^{(1)}+\frac{1}{3} U_{3}^{(1)} \xi_{12}^{(1)}+\frac{1}{6} U_{2}^{(2)} \xi_{11}^{(2)}+\frac{1}{3} U_{3}^{(2)} \xi_{12}^{(2)} \text {, } \\
& \mathbf{D}_{22}=\frac{1}{3} \sum_{k=1}^{n}\left(U_{1}^{q}-U_{2}^{q} \cos 2 \theta_{k}+U_{3}^{q} \cos 4 \theta_{k}\right)\left(z_{k}^{3}-z_{k-1}^{3}\right) \\
& =\frac{1}{3} U_{1}^{(1)} \xi_{15}^{(1)}-\frac{1}{3} U_{2}^{(1)} \xi_{9}^{(1)}+\frac{1}{3} U_{3}^{(1)} \xi_{10}^{(1)}+\frac{1}{3} U_{1}^{(2)} \xi_{15}^{(2)}-\frac{1}{3} U_{2}^{(2)} \xi_{9}^{(2)} \\
& +\frac{1}{3} U_{3}^{(2)} \xi_{10}^{2} \\
& \mathbf{D}_{26}=\mathbf{D}_{62}=\frac{1}{3} \sum_{k=1}^{n}\left[U_{2}^{q}\left(\frac{\sin 2 \theta_{k}}{2}\right)-U_{3}^{q} \sin 4 \theta_{k}\right]\left(z_{k}^{3}-z_{k-1}^{3}\right) \\
& =\frac{1}{6} U_{2}^{(1)} \xi_{11}^{(1)}-\frac{1}{3} U_{3}^{(1)} \xi_{12}^{(1)}+\frac{1}{6} U_{2}^{(2)} \xi_{11}^{(2)}-\frac{1}{3} U_{3}^{(2)} \xi_{12}^{(2)} \text {, } \\
& \mathbf{D}_{66}=\frac{1}{3} \sum_{k=1}^{n}\left(-U_{3}^{q} \cos 4 \theta_{k}+U_{5}^{q}\right)\left(z_{k}^{3}-z_{k-1}^{3}\right) \\
& =-\frac{1}{3} U_{3}^{(1)} \xi_{10}^{(1)}+\frac{1}{3} U_{5}^{(1)} \xi_{15}^{(1)}-\frac{1}{3} U_{3}^{(2)} \xi_{10}^{(2)}+\frac{1}{3} U_{5}^{(2)} \xi_{15}^{(2)} \text {. }
\end{aligned}
$$

To sum up, the stiffness coefficients of the hybrid laminates, which are only related to the material constants and geometric factors, can be expressed as

$$
\left[\begin{array}{l}
\mathbf{A}_{11} \\
\mathbf{A}_{12} \\
\mathbf{A}_{16} \\
\mathbf{A}_{22} \\
\mathbf{A}_{26} \\
\mathbf{A}_{66}
\end{array}\right]=\sum_{q=1,2}\left[\begin{array}{ccccc}
\xi_{13}^{q} & \xi_{1}^{q} & \xi_{2}^{q} & 0 & 0 \\
0 & 0 & -\xi_{2}^{q} & \xi_{13}^{q} & 0 \\
0 & \frac{\xi_{3}^{q}}{2} & \xi_{4}^{q} & 0 & 0 \\
\xi_{13}^{q} & -\xi_{1}^{q} & \xi_{2}^{q} & 0 & 0 \\
0 & \frac{\xi_{3}^{q}}{2} & -\xi_{4}^{q} & 0 & 0 \\
0 & 0 & -\xi_{2}^{q} & 0 & \xi_{13}^{1}
\end{array}\right]\left[\begin{array}{c}
U_{1}^{q} \\
U_{2}^{q} \\
U_{3}^{q} \\
U_{4}^{q} \\
U_{5}^{q}
\end{array}\right],
$$

$$
\left[\begin{array}{l}
\mathbf{B}_{11} \\
\mathbf{B}_{12} \\
\mathbf{B}_{16} \\
\mathbf{B}_{22} \\
\mathbf{B}_{26} \\
\mathbf{B}_{66}
\end{array}\right]=\frac{1}{2} \sum_{q=(1,2)}\left[\begin{array}{ccccc}
\xi_{14}^{q} & \xi_{5}^{q} & \xi_{6}^{q} & 0 & 0 \\
0 & 0 & -\xi_{6}^{q} & \xi_{14}^{q} & 0 \\
0 & \frac{\xi_{7}^{q}}{2} & \xi_{8}^{q} & 0 & 0 \\
\xi_{14}^{q} & -\xi_{5}^{q} & \xi_{6}^{q} & 0 & 0 \\
0 & \frac{\xi_{7}^{q}}{2} & -\xi_{8}^{q} & 0 & 0 \\
0 & 0 & -\xi_{6}^{q} & 0 & \xi_{14}^{q}
\end{array}\right]\left[\begin{array}{c}
U_{1}^{q} \\
U_{2}^{q} \\
U_{3}^{q} \\
U_{4}^{q} \\
U_{5}^{q}
\end{array}\right]
$$

$$
\left[\begin{array}{l}
\mathbf{D}_{11} \\
\mathbf{D}_{12} \\
\mathbf{D}_{16} \\
\mathbf{D}_{22} \\
\mathbf{D}_{26} \\
\mathbf{D}_{66}
\end{array}\right]=\frac{1}{3} \sum_{q=(1,2)}\left[\begin{array}{ccccc}
\xi_{15}^{q} & \xi_{9}^{q} & \xi_{10}^{q} & 0 & 0 \\
0 & 0 & -\xi_{10}^{q} & \xi_{15}^{q} & 0 \\
0 & \frac{\xi_{11}^{q}}{2} & \xi_{12}^{q} & 0 & 0 \\
\xi_{15}^{q} & -\xi_{9}^{q} & \xi_{10}^{q} & 0 & 0 \\
0 & \frac{\xi_{11}^{q}}{2} & -\xi_{12}^{q} & 0 & 0 \\
0 & 0 & -\xi_{10}^{q} & 0 & \xi_{15}^{q}
\end{array}\right]\left[\begin{array}{c}
U_{1}^{q} \\
U_{2}^{q} \\
U_{3}^{q} \\
U_{4}^{q} \\
U_{5}^{q}
\end{array}\right] .
$$

2.2. Thermal Stress and Thermal Moment. The thermal expansion coefficients of the $k$-ply in the entire hybrid laminates are

$$
\begin{gathered}
\left(\alpha_{x}\right)_{k}=\alpha_{1}^{q} \cos ^{2} \theta_{k}+\alpha_{2}^{q} \sin ^{2} \theta_{k}, \\
\left(\alpha_{y}\right)_{k}=\alpha_{1}^{q} \sin ^{2} \theta_{k}+\alpha_{2}^{q} \cos ^{2} \theta_{k}, \\
\left(\alpha_{x y}\right)_{k}=\left(\alpha_{1}^{q}-\alpha_{2}^{q}\right) 2 \sin \theta_{k} \cos \theta_{k},
\end{gathered}
$$

wherein $\alpha_{1}^{q}$ and $\alpha_{2}^{q}(q=$ (1), (2) ) are the thermal expansion coefficients of two different types of lamina. The variation of temperature can be expressed by $\Delta T$, and the thermal stress and thermal moment of laminates are expressed as 


$$
\begin{gathered}
N_{x}^{T}=\sum_{k=1}^{n} \Delta T\left[\left(\bar{Q}_{11}\right)_{k}\left(\alpha_{x}\right)_{k}+\left(\bar{Q}_{12}\right)_{k}\left(\alpha_{y}\right)_{k}+\left(\bar{Q}_{16}\right)_{k}\left(\alpha_{x y}\right)_{k}\right]\left(z_{k}-z_{k-1}\right), \\
N_{y}^{T}=\sum_{k=1}^{n} \Delta T\left[\left(\bar{Q}_{12}\right)_{k}\left(\alpha_{x}\right)_{k}+\left(\bar{Q}_{22}\right)_{k}\left(\alpha_{y}\right)_{k}+\left(\bar{Q}_{26}\right)_{k}\left(\alpha_{x y}\right)_{k}\right]\left(z_{k}-z_{k-1}\right), \\
N_{x y}^{T}=\sum_{k=1}^{n} \Delta T\left[\left(\bar{Q}_{16}\right)_{k}\left(\alpha_{x}\right)_{k}+\left(\bar{Q}_{26}\right)_{k}\left(\alpha_{y}\right)_{k}+\left(\bar{Q}_{66}\right)_{k}\left(\alpha_{x y}\right)_{k}\right]\left(z_{k}-z_{k-1}\right), \\
M_{x}^{T}=\frac{1}{2} \sum_{k=1}^{n} \Delta T\left[\left(\bar{Q}_{11}\right)_{k}\left(\alpha_{x}\right)_{k}+\left(\bar{Q}_{12}\right)_{k}\left(\alpha_{y}\right)_{k}+\left(\bar{Q}_{16}\right)_{k}\left(\alpha_{x y}\right)_{k}\right] \\
\cdot\left(z_{k}^{2}-z_{k-1}^{2}\right), \\
M_{y}^{T}=\frac{1}{2} \sum_{k=1}^{n} \Delta T\left[\left(\bar{Q}_{12}\right)_{k}\left(\alpha_{x}\right)_{k}+\left(\bar{Q}_{22}\right)_{k}\left(\alpha_{y}\right)_{k}+\left(\bar{Q}_{26}\right)_{k}\left(\alpha_{x y}\right)_{k}\right] \\
\cdot\left(z_{k}^{2}-z_{k-1}^{2}\right), \\
M_{x y}^{T}=\frac{1}{2} \sum_{k=1}^{n} \Delta T\left[\left(\bar{Q}_{16}\right)_{k}\left(\alpha_{x}\right)_{k}+\left(\bar{Q}_{26}\right)_{k}\left(\alpha_{y}\right)_{k}+\left(\bar{Q}_{66}\right)_{k}\left(\alpha_{x y}\right)_{k}\right] \\
\cdot\left(z_{k}^{2}-z_{k-1}^{2}\right) .
\end{gathered}
$$

Substituting (1) and (13) into (14) can be used to obtain the expressions of thermal stress, which are only related to the material constants and geometric factors, as shown in

$$
\begin{aligned}
& N_{x}^{T}=\sum_{k=1}^{n} \Delta T\left(\frac{1}{2} U_{1}^{T q}+\frac{1}{2} U_{2}^{T q} \cos 2 \theta_{k}\right)\left(z_{k}-z_{k-1}\right)
\end{aligned}
$$

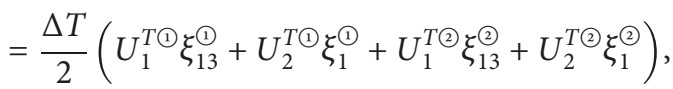

$$
\begin{aligned}
& N_{y}^{T}=\sum_{k=1}^{n} \Delta T\left(\frac{1}{2} U_{1}^{T q}-\frac{1}{2} U_{2}^{T q} \cos 2 \theta_{k}\right)\left(z_{k}-z_{k-1}\right) \\
& =\frac{\Delta T}{2}\left(U_{1}^{T(1)} \xi_{13}^{(1)}-U_{2}^{T(1)} \xi_{1}^{(1)}+U_{1}^{T(} \xi_{13}^{(2}-U_{2}^{T(2)} \xi_{1}^{(2)}\right) \text {, } \\
& N_{x y}^{T}=\frac{\Delta T}{2} \sum_{k=1}^{n} U_{2}^{T q} \sin 2 \theta_{k}\left(z_{k}-z_{k-1}\right) \\
& =\frac{\Delta T}{2}\left(\xi_{3}^{(1)} U_{2}^{T(}+\xi_{3}^{(\supseteq)} U_{2}^{T \odot}\right) .
\end{aligned}
$$

In which, $U_{1}^{T q}$ and $U_{2}^{T q}$ are defined as the thermal invariants of the hybrid laminates, which can be calculated from the thermal expansion coefficients and the invariants of laminates, as shown in

$$
\begin{gathered}
U_{1}^{T q}=\left(\alpha_{1}^{q}+\alpha_{2}^{q}\right)\left(U_{1}^{q}+U_{4}^{q}\right)+\left(\alpha_{1}^{q}-\alpha_{2}^{q}\right) U_{2}^{q}, \\
U_{2}^{T q}=\left(\alpha_{1}^{q}+\alpha_{2}^{q}\right) U_{2}^{q}+\left(\alpha_{1}^{q}-\alpha_{2}^{q}\right)\left(U_{1}^{q}+2 U_{3}^{q}-U_{4}^{q}\right) .
\end{gathered}
$$

Substituting (1) and (13) into (15) can be used to obtain the expressions of the thermal moment, which are only related to the material constants and geometric factors, as shown in

$$
\begin{aligned}
& M_{x}^{T}=\frac{1}{2} \sum_{k=1}^{n} \Delta T\left(\frac{1}{2} U_{1}^{T q}+\frac{1}{2} U_{2}^{T q} \cos 2 \theta_{k}\right)\left(z_{k}^{2}-z_{k-1}^{2}\right) \\
& =\frac{\Delta T}{4}\left(U_{1}^{T(} \xi_{14}^{(1)}+U_{2}^{T(1)} \xi_{5}^{(1)}+U_{1}^{T(2)} \xi_{14}^{(2)}+U_{2}^{T(2)} \xi_{5}^{(2)}\right), \\
& M_{y}^{T}=\frac{1}{2} \sum_{k=1}^{n} \Delta T\left(\frac{1}{2} U_{1}^{T q}-\frac{1}{2} U_{2}^{T q} \cos 2 \theta_{k}\right)\left(z_{k}^{2}-z_{k-1}^{2}\right) \\
& =\frac{\Delta T}{4}\left(U_{1}^{T\left(\xi_{14}^{(1}\right.}-U_{2}^{T()_{5}^{(1)}}+U_{1}^{T \odot} \xi_{14}^{(2}-U_{2}^{T \odot} \xi_{5}^{(}\right), \\
& M_{x y}^{T}=\frac{\Delta T}{4} \sum_{k=1}^{n} U_{2}^{T q} \sin 2 \theta_{k}\left(z_{k}-z_{k-1}\right) \\
& =\frac{\Delta T}{4}\left(\xi_{7}^{(1)} U_{2}^{T(1)}+\xi_{7}^{\circledR} U_{2}^{T(2)}\right) .
\end{aligned}
$$

To sum up, the thermal stress and thermal moment of the hybrid laminates, which are only related to the thermal invariants and geometric factors, can be expressed as

$$
\begin{aligned}
& {\left[\begin{array}{c}
N_{x}^{T} \\
N_{y}^{T} \\
N_{x y}^{T}
\end{array}\right]=\frac{\Delta T}{2} \sum_{q=(1), 2}\left[\begin{array}{c}
U_{1}^{T q} \xi_{13}^{q}+U_{2}^{T q} \xi_{1}^{q} \\
U_{1}^{T q} \xi_{13}^{q}-U_{2}^{T q} \xi_{1}^{q} \\
U_{2}^{T q} \xi_{3}^{q}
\end{array}\right],} \\
& {\left[\begin{array}{l}
M_{x}^{T} \\
M_{y}^{T} \\
M_{x y}^{T}
\end{array}\right]=\frac{\Delta T}{4} \sum_{q=(1), 2}\left[\begin{array}{c}
U_{1}^{T q} \xi_{14}^{q}+U_{2}^{T q} \xi_{5}^{q} \\
U_{1}^{T q} \xi_{14}^{q}-U_{2}^{T q} \xi_{5}^{q} \\
U_{2}^{T q} \xi_{7}^{q}
\end{array}\right] .}
\end{aligned}
$$

\section{The Hybrid $A_{F} B_{0} D_{S}$ Laminates with Immunity to HTSD}

3.1. Necessary and Sufficient Conditions. From (10), (11), and (12), for matrices $\mathbf{A}, \mathbf{B}$, and $\mathbf{D}$, respectively, can we see that the stiffness coefficients of laminates are only related to geometrical factors and material constants. In order to make the designed laminates suitable for all materials, the necessary and sufficient conditions of geometric factors for the hybrid $\mathbf{A}_{F} \mathbf{B}_{0} \mathbf{D}_{S}$ laminates with immunity to HTSD will be derived in this section. The stiffness coefficients of hybrid $\mathbf{A}_{F} \mathbf{B}_{0} \mathbf{D}_{S}$ laminates should meet the following relationships [2].

$$
\begin{aligned}
\mathbf{B}_{11}=\mathbf{B}_{12}=\mathbf{B}_{16} & =\mathbf{B}_{22}=\mathbf{B}_{26}=\mathbf{B}_{66}=0, \\
\mathbf{D}_{16} & =\mathbf{D}_{26}=0 .
\end{aligned}
$$

Substituting (11) and (12) into Eq. (21) can be used to obtain the necessary and sufficient conditions of geometric factors for the hybrid $\mathbf{A}_{F} \mathbf{B}_{0} \mathbf{D}_{S}$ laminates.

$$
\begin{aligned}
& \xi_{5}^{(1)}=\xi_{6}^{(1)}=\xi_{7}^{(1)}=\xi_{8}^{(1)}=\xi_{11}^{(1)}=\xi_{12}^{(1)}=\xi_{14}^{(1)}=0, \\
& \xi_{5}^{(2)}=\xi_{6}^{(2)}=\xi_{7}^{(2)}=\xi_{8}^{(2)}=\xi_{11}^{(2)}=\xi_{12}^{(2)}=\xi_{14}^{(2)}=0 .
\end{aligned}
$$

In order to ensure that the hybrid $\mathbf{A}_{F} \mathbf{B}_{0} \mathbf{D}_{S}$ laminates will not cause hygrothermal shear distortion, its thermal shear strain should meet following relationship. 


$$
\gamma_{x y}^{T}=0
$$

Moreover, the connection between thermal stress $N^{T}$ and thermal shear strain $\gamma_{x y}^{T}$ of $\mathbf{A}_{F} \mathbf{B}_{0} \mathbf{D}_{S}$ hybrid laminates is

$$
\left\{\begin{array}{c}
\varepsilon_{x}^{T} \\
\varepsilon_{y}^{T} \\
\gamma_{x y}^{T}
\end{array}\right\}=\left[\begin{array}{lll}
\mathbf{A}_{11} & \mathbf{A}_{12} & \mathbf{A}_{16} \\
\mathbf{A}_{12} & \mathbf{A}_{22} & \mathbf{A}_{26} \\
\mathbf{A}_{16} & \mathbf{A}_{26} & \mathbf{A}_{66}
\end{array}\right]^{-1}\left\{\begin{array}{c}
N_{x}^{T} \\
N_{y}^{T} \\
N_{x y}^{T}
\end{array}\right\}=\frac{\mathbf{A}^{*}}{|\mathbf{A}|}\left\{\begin{array}{c}
N_{x}^{T} \\
N_{y}^{T} \\
N_{x y}^{T}
\end{array}\right\},
$$

where

$$
\mathbf{A}^{*}=\left[\begin{array}{ccc}
\mathbf{A}_{22} \mathbf{A}_{66}-\mathbf{A}_{26}^{2} & \mathbf{A}_{16} \mathbf{A}_{26}-\mathbf{A}_{12} \mathbf{A}_{66} & \mathbf{A}_{12} \mathbf{A}_{26}-\mathbf{A}_{16} \mathbf{A}_{22} \\
\mathbf{A}_{16} \mathbf{A}_{26}-\mathbf{A}_{12} \mathbf{A}_{66} & \mathbf{A}_{11} \mathbf{A}_{66}-\mathbf{A}_{16}^{2} & \mathbf{A}_{16} \mathbf{A}_{16}-\mathbf{A}_{11} \mathbf{A}_{26} \\
\mathbf{A}_{12} \mathbf{A}_{26}-\mathbf{A}_{16} \mathbf{A}_{22} & \mathbf{A}_{16} \mathbf{A}_{16}-\mathbf{A}_{11} \mathbf{A}_{26} & \mathbf{A}_{11} \mathbf{A}_{22}-\mathbf{A}_{12}^{2}
\end{array}\right] .
$$

Substituting (25) into (24) can be used to obtain the expression of thermal shear strain of the hybrid $\mathbf{A}_{F} \mathbf{B}_{0} \mathbf{D}_{S}$ laminates.

$$
\begin{aligned}
\gamma_{x y}^{T}=\frac{1}{|\mathbf{A}|} & {\left[\left(\mathbf{A}_{12} \mathbf{A}_{26}-\mathbf{A}_{16} \mathbf{A}_{22}\right) N_{x}^{T}+\left(\mathbf{A}_{12} \mathbf{A}_{16}-\mathbf{A}_{11} \mathbf{A}_{26}\right) N_{y}^{T}\right.} \\
& \left.+\left(\mathbf{A}_{11} \mathbf{A}_{22}-\mathbf{A}_{12}^{2}\right) N_{x y}^{T}\right] .
\end{aligned}
$$

Inserting (26) into (23) gives

$$
\begin{aligned}
& \left(\mathbf{A}_{12} \mathbf{A}_{26}-\mathbf{A}_{16} \mathbf{A}_{22}\right) N_{x}^{T}+\left(\mathbf{A}_{12} \mathbf{A}_{16}-\mathbf{A}_{11} \mathbf{A}_{26}\right) N_{y}^{T} \\
& +\left(\mathbf{A}_{11} \mathbf{A}_{22}-\mathbf{A}_{12}^{2}\right) N_{x y}^{T}=0 .
\end{aligned}
$$

Inserting (19) into (27) and simplifying gives

$$
\begin{aligned}
& {\left[\left(\mathbf{A}_{12} \mathbf{A}_{26}-\mathbf{A}_{16} \mathbf{A}_{22}\right)+\left(\mathbf{A}_{12} \mathbf{A}_{16}-\mathbf{A}_{11} \mathbf{A}_{26}\right)\right]} \\
& \quad \sum_{q=1,(2)} U_{1}^{T q} \xi_{13}^{q}+\left[\left(\mathbf{A}_{12} \mathbf{A}_{26}-\mathbf{A}_{16} \mathbf{A}_{22}\right)-\left(\mathbf{A}_{12} \mathbf{A}_{16}-\mathbf{A}_{11} \mathbf{A}_{26}\right)\right] \\
& \quad \sum_{q=\text { (1),2 }} U_{2}^{T q} \xi_{1}^{q}+\left(\mathbf{A}_{11} \mathbf{A}_{22}-\mathbf{A}_{12}^{2}\right) \sum_{q=\text { (1), (2) }} U_{2}^{T q} \xi_{3}^{q}=0 .
\end{aligned}
$$

If (28) is constantly established, the previous coefficient of thermal material constant $U_{1}^{T q}$ and $U_{2}^{T q}(q=$ (1), (2)) must be zero.

$$
\left[\left(\mathbf{A}_{12} \mathbf{A}_{26}-\mathbf{A}_{16} \mathbf{A}_{22}\right)+\left(\mathbf{A}_{12} \mathbf{A}_{16}-\mathbf{A}_{11} \mathbf{A}_{26}\right)\right] \xi_{13}^{q}=0(q=\text { (1), (2) })
$$

$$
\begin{aligned}
& {\left[\left(\mathbf{A}_{12} \mathbf{A}_{26}-\mathbf{A}_{16} \mathbf{A}_{22}\right)-\left(\mathbf{A}_{12} \mathbf{A}_{16}-\mathbf{A}_{11} \mathbf{A}_{26}\right)\right] \xi_{1}^{q}} \\
& +\left(\mathbf{A}_{11} \mathbf{A}_{22}-\mathbf{A}_{12}^{2}\right) \xi_{3}^{q}=0(q=\text { (1), (2) }) .
\end{aligned}
$$

According to (30) can we find out that

$$
\xi_{1}^{q}=\xi_{3}^{q}=0(q=\text { (1), (2) }) \text {. }
$$

Inserting (31) into (10) gives

$$
\left[\begin{array}{l}
\mathbf{A}_{11} \\
\mathbf{A}_{12} \\
\mathbf{A}_{16} \\
\mathbf{A}_{22} \\
\mathbf{A}_{26} \\
\mathbf{A}_{66}
\end{array}\right]=\sum_{q=(1,2)}\left[\begin{array}{ccccc}
\xi_{13}^{q} & 0 & \xi_{2}^{q} & 0 & 0 \\
0 & 0 & -\xi_{2}^{q} & \xi_{13}^{q} & 0 \\
0 & 0 & \xi_{4}^{q} & 0 & 0 \\
\xi_{13}^{q} & 0 & \xi_{2}^{q} & 0 & 0 \\
0 & 0 & -\xi_{4}^{q} & 0 & 0 \\
0 & 0 & -\xi_{2}^{q} & 0 & \xi_{13}^{q}
\end{array}\right]\left[\begin{array}{c}
U_{1}^{q} \\
U_{2}^{q} \\
U_{3}^{q} \\
U_{4}^{q} \\
U_{5}^{q}
\end{array}\right] .
$$

Then inserting (32) into (29) further gives

$$
\begin{aligned}
& \left(\mathbf{A}_{12} \mathbf{A}_{26}-\mathbf{A}_{16} \mathbf{A}_{22}\right)+\left(\mathbf{A}_{12} \mathbf{A}_{16}-\mathbf{A}_{11} \mathbf{A}_{26}\right) \\
& =\left(\mathbf{A}_{26}+\mathbf{A}_{16}\right)\left(\mathbf{A}_{12}-\mathbf{A}_{11}\right) .
\end{aligned}
$$

Furthermore, because of $\left(\mathbf{A}_{26}+\mathbf{A}_{16}\right)=0$ and $\xi_{13}^{q} \neq 0$, (29) is also established. Considering that if $\xi_{4}^{q}=0, \mathbf{A}_{16}$ and $\mathbf{A}_{26}$ of laminates are both zero and the laminates will not have extension-shear-coupled effect. Therefore, geometric factor $\xi_{4}^{q}$ cannot be zero; thus, the necessary and sufficient conditions for "with immunity to HTSD" can be expressed as

$$
\xi_{1}^{(1)}=\xi_{3}^{(1)}=\xi_{1}^{(2)}=\xi_{3}^{(2)}=0, \xi_{4}^{(1)} \neq 0, \xi_{4}^{(2)} \neq 0 .
$$

In summary, the necessary and sufficient conditions of the hybrid $\mathbf{A}_{F} \mathbf{B}_{0} \mathbf{D}_{S}$ laminates with immunity to HTSD can be expressed as

$$
\begin{aligned}
& \xi_{1}^{(1)}=\xi_{3}^{(1)}=\xi_{1}^{(2)}=\xi_{3}^{(2)}=0, \xi_{4}^{(1)} \neq 0, \xi_{4}^{(2)} \neq 0, \\
& \xi_{5}^{(1)}=\xi_{6}^{(1)}=\xi_{7}^{(1)}=\xi_{8}^{(1)}=\xi_{11}^{(1)}=\xi_{12}^{(1)}=\xi_{14}^{(1)}=0, \\
& \xi_{5}^{(2)}=\xi_{6}^{(2)}=\xi_{7}^{(2)}=\xi_{8}^{(2)}=\xi_{11}^{(2)}=\xi_{12}^{(2)}=\xi_{14}^{(2)}=0 .
\end{aligned}
$$

3.2. Curing Deformation. Considering that the curing deformation phenomenon will occur during the process of molding, which may bring about deformation difference between the expected design shape and the free shape after taking off the model at room temperature, now, the thermal strain of the hybrid $\mathbf{A}_{F} \mathbf{B}_{0} \mathbf{D}_{S}$ laminates with immunity to HTSD will be derived by the necessary and sufficient conditions during the process of curing deformation.

Due to the similarity of influence between humidity changes and temperature changes on composite materials, it only needs to replace the thermal expansion coefficients by humidity expansion coefficients. In order to simplify the analysis and derivation process, the analysis for hygrothermal distortion contains the thermal effect merely.

Substituting (25) into (24) can be used to obtain the expressions of thermal strain of the hybrid $\mathbf{A}_{F} \mathbf{B}_{0} \mathbf{D}_{S}$ laminates with immunity to HTSD. 


$$
\begin{aligned}
\varepsilon_{x}^{T}=\frac{1}{|\mathbf{A}|} & {\left[\left(\mathbf{A}_{22} \mathbf{A}_{66}-\mathbf{A}_{26}^{2}\right) N_{x}^{T}+\left(\mathbf{A}_{16} \mathbf{A}_{26}-\mathbf{A}_{12} \mathbf{A}_{66}\right) N_{y}^{T}\right.} \\
& \left.+\left(\mathbf{A}_{12} \mathbf{A}_{26}-\mathbf{A}_{16} \mathbf{A}_{22}\right) N_{x y}^{T}\right], \\
\varepsilon_{y}^{T}=\frac{1}{|\mathbf{A}|}[ & \left(\mathbf{A}_{16} \mathbf{A}_{26}-\mathbf{A}_{12} \mathbf{A}_{66}\right) N_{x}^{T}+\left(\mathbf{A}_{11} \mathbf{A}_{66}-\mathbf{A}_{16}^{2}\right) N_{y}^{T} \\
& \left.+\left(\mathbf{A}_{16} \mathbf{A}_{16}-\mathbf{A}_{11} \mathbf{A}_{26}\right) N_{x y}^{T}\right] .
\end{aligned}
$$

Substituting (35) into (19) can give the expressions of thermal stress of the hybrid $\mathbf{A}_{F} \mathbf{B}_{0} \mathbf{D}_{S}$ laminates with immunity to HTSD.

$$
\left[\begin{array}{c}
N_{x}^{T} \\
N_{y}^{T} \\
N_{x y}^{T}
\end{array}\right]=\frac{\Delta T}{2}\left[\begin{array}{c}
U_{1}^{T(1)} \xi_{13}^{(1)} \\
U_{1}^{T(1)} \xi_{13}^{(1)} \\
0
\end{array}\right]+\frac{\Delta T}{2}\left[\begin{array}{c}
U_{1}^{T(2)} \xi_{13}^{(2)} \\
U_{1}^{T(2)} \xi_{13}^{(2)} \\
0
\end{array}\right] .
$$

Inserting (10), (35), and (37) into (36) gives

$$
\begin{aligned}
& \varepsilon_{x}^{T}=\varepsilon_{y}^{T}=\frac{\Delta T}{2}\left[\mathbf{A}_{16}\left(\mathbf{A}_{26}-\mathbf{A}_{16}\right)+\mathbf{A}_{66}\left(\mathbf{A}_{11}-\mathbf{A}_{12}\right)\right] \\
& \cdot\left(U_{1}^{T(1)} \xi_{13}^{(1)}+U_{1}^{T(2)} \xi_{13}^{(2)}\right) \text {. }
\end{aligned}
$$

Therefore, the thermal strains of two main directions of the hybrid $\mathbf{A}_{F} \mathbf{B}_{0} \mathbf{D}_{S}$ laminates with immunity to HTSD are equal to each other and the value of the thermal strain is not only related to the material constants and temperature variation but also changing with geometric factors $\xi_{2}^{q}, \xi_{4}^{q}$, and $\xi_{13}^{q}$.

\section{Optimized Design of Laminates}

Considering that the extension-shear-coupled effect of hybrid $\mathbf{A}_{F} \mathbf{B}_{0} \mathbf{D}_{S}$ laminates with immunity to HTSD is the main performance index, the maximum extension-shearcoupled effect is therefore the major objective. Regarding the ply materials and ply angles of each lamina as variables, take the ply materials and ply angles as the optimized design variables. The optimized constraint conditions can be obtained by (35), which ensures that the final optimized laminates are hybrid $\mathbf{A}_{F} \mathbf{B}_{0} \mathbf{D}_{S}$ laminates with immunity to HTSD. Take the flexibility coefficient $a_{16}$ as the parameter to test the extension-shear-coupled effect of laminates, which can be obtained by inverting the stiffness matrix of laminates, as shown in (39).

$$
\left[\begin{array}{llllll}
a_{11} & a_{12} & a_{16} & b_{11} & b_{12} & b_{16} \\
a_{12} & a_{22} & a_{26} & b_{12} & b_{22} & b_{26} \\
a_{16} & a_{26} & a_{66} & b_{16} & b_{26} & b_{66} \\
b_{11} & b_{12} & b_{16} & d_{11} & d_{12} & d_{16} \\
b_{12} & b_{22} & b_{26} & d_{12} & d_{22} & d_{26} \\
b_{16} & b_{26} & b_{66} & d_{16} & d_{26} & d_{66}
\end{array}\right]=\left[\begin{array}{llllll}
\mathbf{A}_{11} & \mathbf{A}_{12} & \mathbf{A}_{16} & \mathbf{B}_{11} & \mathbf{B}_{12} & \mathbf{B}_{16} \\
\mathbf{A}_{12} & \mathbf{A}_{22} & \mathbf{A}_{26} & \mathbf{B}_{12} & \mathbf{B}_{22} & \mathbf{B}_{26} \\
\mathbf{A}_{16} & \mathbf{A}_{26} & \mathbf{A}_{66} & \mathbf{B}_{16} & \mathbf{B}_{26} & \mathbf{B}_{66} \\
\mathbf{B}_{11} & \mathbf{B}_{12} & \mathbf{B}_{16} & \mathbf{D}_{11} & \mathbf{D}_{12} & \mathbf{D}_{16} \\
\mathbf{B}_{12} & \mathbf{B}_{22} & \mathbf{B}_{26} & \mathbf{D}_{12} & \mathbf{D}_{22} & \mathbf{D}_{26} \\
\mathbf{B}_{16} & \mathbf{B}_{26} & \mathbf{B}_{66} & \mathbf{D}_{16} & \mathbf{D}_{26} & s_{66}
\end{array}\right]^{-}
$$

TABLE 1: Material properties of carbon fiber lamina and glass fiber lamina.

\begin{tabular}{lccc}
\hline Performance parameters & & $\begin{array}{c}\text { Carbon fiber } \\
\text { lamina }\end{array}$ & $\begin{array}{c}\text { Glass fiber } \\
\text { lamina }\end{array}$ \\
\hline Elastic modulus (GPa) & $E_{1}$ & 181.0 & 38.6 \\
Shear modulus (GPa) & $E_{2}$ & 10.2 & 8.3 \\
Poisson's ratio & $G_{12}$ & 7.2 & 4.14 \\
Thickness (mm) & $v_{12}$ & 0.28 & 0.26 \\
Thermal expansion & $t$ & 0.1 & 0.1 \\
coefficient $\left(\mu /^{\circ} \mathrm{C}\right)$ & $\alpha_{1}$ & -0.1 & 8.6 \\
\hline
\end{tabular}

However, taking into account the actual engineering demands, which should be combined with the good comprehensive properties of carbon fiber composites and the low cost of glass fiber composites, the proportion of two kinds of material must have a minimum requirement. On the one hand, if the proportion of carbon fiber composites is too small, the structural stability of the hybrid laminates cannot be guaranteed. On the other hand, if the proportion of glass fiber composites is too small, the cost reduction effect is not obvious. Therefore, in this paper, the minimum proportions of two types of material are both set as 30\% and the mathematical model of optimization problem can be formulated as

$$
\begin{aligned}
& \min \quad F\left(q_{1}, q_{2}, \ldots, q_{n}, \theta_{1}, \theta_{2}, \ldots, \theta_{n}\right)=-\left|a_{16}\right|, \\
& \text { s.t. }\left\{\begin{array}{l}
1.3 n \leq \sum_{i=1}^{n} q_{k} \leq 1.7 n, \\
-90^{\circ}<\theta_{k} \leq 90^{\circ}, \quad k=1,2, \ldots, n, \\
\sum_{q_{k}=1} \cos 2 \theta_{k}\left(z_{k}-z_{k-1}\right)=\sum_{q_{k}=2} \cos 2 \theta_{k}\left(z_{k}-z_{k-1}\right)=0, \\
\sum_{q_{k}=1} \sin 2 \theta_{k}\left(z_{k}-z_{k-1}\right)=\sum_{q_{k}=2} \sin 2 \theta_{k}\left(z_{k}-z_{k-1}\right)=0, \\
\sum_{q_{k}=1} \cos 2 \theta_{k}\left(z_{k}^{2}-z_{k-1}^{2}\right)=\sum_{q_{k}=2} \cos 2 \theta_{k}\left(z_{k}^{2}-z_{k-1}^{2}\right)=0, \\
\sum_{q_{k}=1} \cos 4 \theta_{k}\left(z_{k}^{2}-z_{k-1}^{2}\right)=\sum_{q_{k}=2} \cos 4 \theta_{k}\left(z_{k}^{2}-z_{k-1}^{2}\right)=0, \\
\sum_{q_{k}=1} \sin 2 \theta_{k}\left(z_{k}^{2}-z_{k-1}^{2}\right)=\sum_{q_{k}=2} \sin 2 \theta_{k}\left(z_{k}^{2}-z_{k-1}^{2}\right)=0, \\
\sum_{q_{k}=1} \sin 4 \theta_{k}\left(z_{k}^{2}-z_{k-1}^{2}\right)=\sum_{q_{k}=2} \sin 4 \theta_{k}\left(z_{k}^{2}-z_{k-1}^{2}\right)=0, \\
\sum_{q_{k}=1} \sin 2 \theta_{k}\left(z_{k}^{3}-z_{k-1}^{3}\right)=\sum_{q_{k}=2} \sin 2 \theta_{k}\left(z_{k}^{3}-z_{k-1}^{3}\right)=0, \\
\sum_{q_{k}=1} \sin 4 \theta_{k}\left(z_{k}^{3}-z_{k-1}^{3}\right)=\sum_{q_{k}=2} \sin 4 \theta_{k}\left(z_{k}^{3}-z_{k-1}^{3}\right)=0, \\
\sum_{q_{k}=1}\left(z_{k}^{2}-z_{k-1}^{2}\right)=\sum\left(z_{k}^{2}-z_{k-1}^{2}\right)=0, \\
\sum_{q_{k}=2} \sin 4 \theta_{k}\left(z_{k}-z_{k-1}\right) \neq 0, \sum_{q_{k}=2} \sin 4 \theta_{k}\left(z_{k}-z_{k-1}\right) \neq 0 .
\end{array}\right.
\end{aligned}
$$


TABle 2: Hybrid $\mathbf{A}_{F} \mathbf{B}_{0} \mathbf{D}_{S}$ laminates with immunity to HTSD.

\begin{tabular}{|c|c|c|}
\hline Number of plies & Stacking sequence $/^{\circ}$ & $\left|a_{16}\right| / \mathrm{m} \cdot \mathrm{N}^{-1}$ \\
\hline $1-11$ & No feasible solution & \\
\hline 12 & {$\left[1.2623_{\mathrm{c}} /-88.1563_{\mathrm{c}} / 86.2219_{\mathrm{c}} /-4.7557_{\mathrm{c}} / 90_{\mathrm{gl}} / 0_{\mathrm{gl}} / 0_{\mathrm{gl}} / 90_{\mathrm{gl}} / 85.2442_{\mathrm{c}} /-3.7780_{\mathrm{c}} / 1.8436_{\mathrm{c}} /-88.7376_{\mathrm{c}}\right]_{T}$} & $2.28 \times 10^{-9}$ \\
\hline 13 & {$\left[78.6287_{\mathrm{c}} / 2.5518_{\mathrm{c}} /-62.5528_{\mathrm{c}} /-4.4571_{\mathrm{c}} / 0_{\mathrm{gl}} / 90_{\mathrm{gl}} / 57.6811_{\mathrm{c}} / 90_{\mathrm{gl}} / 0_{\mathrm{gl}} /-22.1785_{\mathrm{c}} / 74.0623_{\mathrm{c}} /-77.5022_{\mathrm{c}} / 5.4030_{\mathrm{c}}\right]_{T}$} & $2.15 \times 10^{-9}$ \\
\hline 14 & $\begin{array}{c}{\left[-82.7030_{\mathrm{c}} / 3.4955_{\mathrm{c}} /-9.5277_{\mathrm{c}} / 68.6342_{\mathrm{c}} /-87.1720_{\mathrm{gl}} /-4.8666_{\mathrm{gl}} / 44.7270_{\mathrm{gl}} /-44.7270_{\mathrm{gl}} / 4.8666_{\mathrm{gl}} / 87.1720_{\mathrm{gl}} /\right.} \\
\left.-86.6975_{\mathrm{c}} /-18.9236_{\mathrm{c}} / 84.4406_{\mathrm{c}} / 9.1474_{\mathrm{c}}\right]_{T}\end{array}$ & $2.62 \times 10^{-9}$ \\
\hline 15 & $\begin{array}{c}{\left[13.7114_{\mathrm{c}} / 79.6085_{\mathrm{c}} /-25.7171_{\mathrm{c}} /-74.2581_{\mathrm{c}} / 85.3581_{\mathrm{gl}} / 7.4729_{\mathrm{gl}} /-44.4060_{\mathrm{gl}} / 15.8105_{\mathrm{c}} / 44.4060_{\mathrm{gl}} /-7.4729_{\mathrm{gl}} /\right.} \\
\left.-85.3581_{\mathrm{gl}} /-88.3194_{\mathrm{c}} /-87.3471_{\mathrm{c}} / 24.6320_{\mathrm{c}} /-19.9766_{\mathrm{c}}\right]_{T}\end{array}$ & $1.68 \times 10^{-9}$ \\
\hline 16 & $\begin{array}{c}{\left[-82.2580_{\mathrm{c}} / 3.4541_{\mathrm{c}} /-7.8035_{\mathrm{c}} / 71.9029_{\mathrm{c}} / 0.0178_{\mathrm{gl}} /-0.0360_{\mathrm{gl}} /-89.9848_{\mathrm{gg}} / 89.9417_{\mathrm{gg}} /-89.9861_{\mathrm{gl}} / 89.9911_{\mathrm{gl}} /\right.} \\
\left.-0.0421_{\mathrm{gl}} / 0.0222_{\mathrm{gl}} /-86.5215_{\mathrm{c}} /-16.3448_{\mathrm{c}} / 84.9501_{\mathrm{c}} / 9.0614_{\mathrm{c}}\right]_{T}\end{array}$ & $2.10 \times 10^{-9}$ \\
\hline 17 & $\begin{array}{c}{\left[-86.7602_{\mathrm{c}} / 86.2949_{\mathrm{c}} /-3.1492_{\mathrm{c}} / 3.7823_{\mathrm{c}} /-4.3788_{\mathrm{gl}} / 35.9403_{\mathrm{gl}} /-50.2104_{\mathrm{gl}} / 89.9014_{\mathrm{gl}} /-72.6480_{\mathrm{gl}} / 41.0040_{\mathrm{gl}} /\right.} \\
\left.-73.7900_{\mathrm{gl}} / 27.9322_{\mathrm{gl}} /-16.0242_{\mathrm{gl}} /-3.7823_{\mathrm{c}} / 3.1492_{\mathrm{c}} /-86.2949_{\mathrm{c}} / 86.7602_{\mathrm{c}}\right]_{T}\end{array}$ & $8.26 \times 10^{-10}$ \\
\hline 18 & $\begin{array}{c}{\left[-0.0130_{\mathrm{gl}} /-89.9908_{\mathrm{gl}} / 0.0171_{\mathrm{gl}} / 89.9876_{\mathrm{gl}} /-80.5781_{\mathrm{c}} / 79.3162_{\mathrm{c}} / 7.2684_{\mathrm{c}} /-20.7364_{\mathrm{c}} / 13.9886_{\mathrm{c}} / 12.0891_{\mathrm{c}} /\right.} \\
\left.-86.7465_{\mathrm{c}} /-66.9711_{\mathrm{c}} / 0.1373_{\mathrm{c}} / 80.6754_{\mathrm{c}} /-89.9869_{\mathrm{gl}} /-0.0163_{\mathrm{gl}} / 89.9904_{\mathrm{gl}} / 0.0125_{\mathrm{gl}}\right]_{T}\end{array}$ & $1.93 \times 10^{-9}$ \\
\hline 19 & $\begin{array}{c}{\left[-3.8195_{\mathrm{c}} /-1.6516_{\mathrm{c}} /-87.2690_{\mathrm{c}} /-86.3382_{\mathrm{c}} / 39.6427_{\mathrm{c}} /-81.7483_{\mathrm{c}} /-0.0004_{\mathrm{gg}} /-89.9971_{\mathrm{gl}} / 89.9928_{\mathrm{gl}} /-14.8789_{\mathrm{c}} /\right.} \\
\left.\quad-0.0071_{\mathrm{gl}} / 0.0028_{\mathrm{gl}} / 89.9995_{\mathrm{gl}} / 25.4403_{\mathrm{c}} /-72.0969_{\mathrm{c}} /-83.9994_{\mathrm{c}} /-0.8820_{\mathrm{c}} / 79.5202_{\mathrm{c}} /-2.8331_{\mathrm{c}}\right]_{T}\end{array}$ & $1.93 \times 10^{-9}$ \\
\hline 20 & $\begin{array}{l}{\left[88.5760_{\mathrm{gl}} / 38.9195_{\mathrm{gl}} /-76.4222_{\mathrm{gl}} /-17.7876_{\mathrm{gl}} /-8.6194_{\mathrm{gl}} / 12.7407_{\mathrm{c}} /-21.3605_{\mathrm{c}} /-87.3614_{\mathrm{c}} / 64.3117_{\mathrm{c}} /-30.3418_{\mathrm{c}} /\right.} \\
\left.\quad-87.4610_{\mathrm{c}} / 48.9897_{\mathrm{c}} / 89.9981_{\mathrm{c}} /-24.1090_{\mathrm{c}} / 7.9710_{\mathrm{c}} / 14.9679_{\mathrm{gl}} / 69.9391_{\mathrm{gl}} / 0.3258_{\mathrm{gl}} /-50.8020_{\mathrm{gl}} / 86.2064_{\mathrm{gl}}\right]_{T}\end{array}$ & $2.20 \times 10^{-9}$ \\
\hline
\end{tabular}

TABLE 3: Maximum extension-shear-coupled effect of two single-composite laminates.

\begin{tabular}{|c|c|c|c|c|c|c|c|c|c|}
\hline Number of plies & 12 & 13 & 14 & 15 & 16 & 17 & 18 & 19 & 20 \\
\hline Carbon fiber laminates $\left(10^{-9} \mathrm{~m} \cdot \mathrm{N}^{-1}\right)$ & 2.80 & 2.71 & 2.36 & 2.10 & 3.01 & 0.809 & 1.93 & 2.19 & 1.26 \\
\hline Glass fiber laminates $\left(10^{-9} \mathrm{~m} \cdot \mathrm{N}^{-1}\right)$ & 12.3 & 13.0 & 11.0 & 8.76 & 11.0 & 10.4 & 9.40 & 8.53 & 7.59 \\
\hline
\end{tabular}

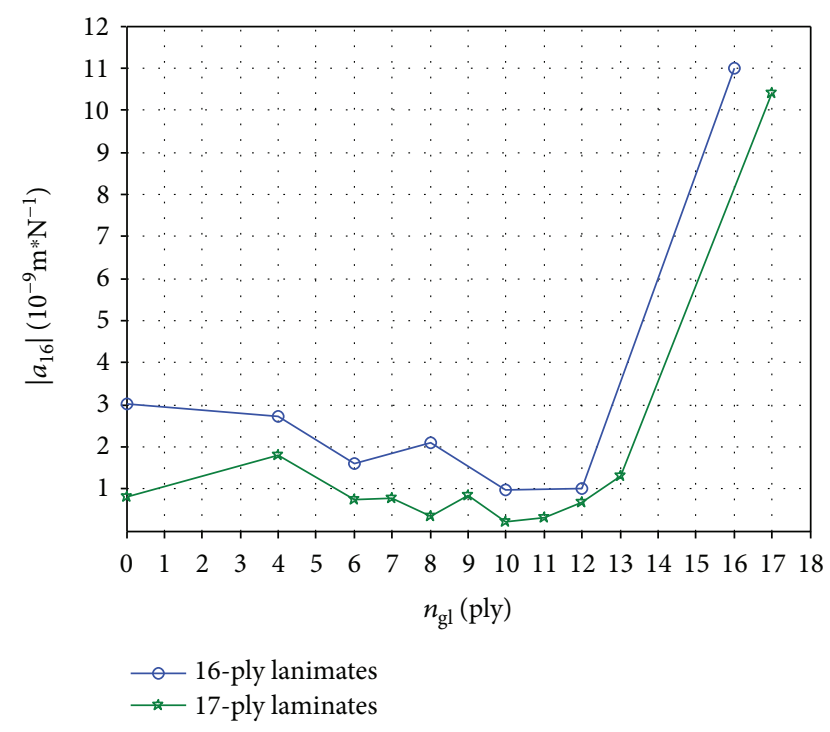

Figure 2: Maximal extension-shear-coupled effect of hybrid laminates with different proportions of two materials.

Wherein, $q_{k}=1,2(k=1,2, \ldots, n)$ represents the values of " $q$ " for the $k$-ply, $q_{k}=1$ shows that the lamina corresponds to the type (1) lamina, and $q_{k}=2$ shows that the lamina corresponds to the type (2) lamina. If $\sum_{k=1}^{n} q_{k}=n$, it means that all lamina are made of type (1) material, and similarly, if $\sum_{k=1}^{n} q_{k}$ $=2 n$, it means that all lamina are made of type (2) material. $\theta_{k}$ represents the paving angle of the $k$-ply, whose initial value is selected randomly from the range of $\left[-90^{\circ}, 90^{\circ}\right]$.

The improved differential evolution algorithm DE_CMSBHS is used to optimize this problem. Because of the strong constraints of optimization problems, which have 18 equality constraints and 2 inequality constraints, the penalty function is applied to deal with the constraints. The type (1) lamina and type (2) lamina are made up of carbon fiber composites and glass fiber composites, respectively, and the corresponding material parameters are shown in Table 1.

Table 2 shows the 12-20-ply hybrid $\mathbf{A}_{F} \mathbf{B}_{0} \mathbf{D}_{S}$ laminates with immunity to HTSD which are optimized by using DE_CMSBHS algorithm to maximize the extension-shearcoupled effect. The subscripts "c" and "gl" in the table indicate that the lamina is made of carbon fiber composites and glass fiber composites, respectively. As can be seen from the table, (1) there is no hybrid $\mathbf{A}_{F} \mathbf{B}_{0} \mathbf{D}_{S}$ laminates with immunity to HTSD for 1-11-ply laminates; (2) for 12-20-ply hybrid $\mathbf{A}_{F} \mathbf{B}_{0} \mathbf{D}_{S}$ laminates with immunity to HTSD, the proportions of two kinds of lamina are both not less than 30\%; and (3) for optimized laminates with the maximum extension-shear-coupled effect, paving materials are symmetrical about the geometric middle plane and have a relatively uniform distribution, which is the one in the middle and the other on both sides.

In order to reflect the influence of the hybrid form on the extension-shear-coupled effect of laminates more intuitively, this paper further optimizes the extension-shear-coupled 


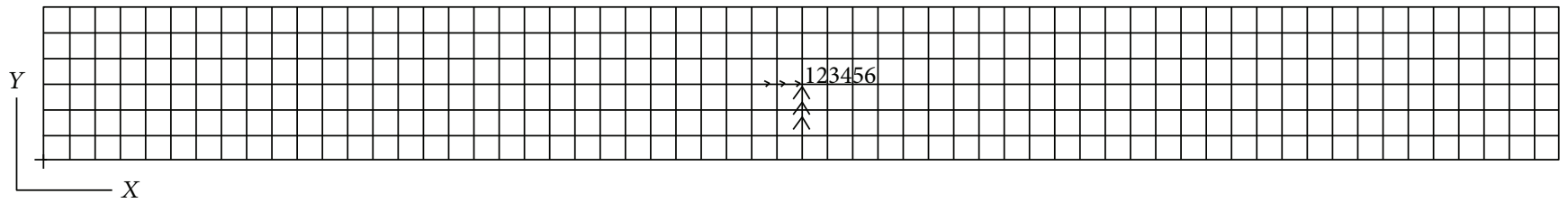

Figure 3: Finite element model of the laminates for hygrothermal effect verification.

TABle 4: Simulation results of hybrid $\mathbf{A}_{F} \mathbf{B}_{0} \mathbf{D}_{S}$ laminates on thermal distortion.

\begin{tabular}{lcccccc}
\hline Type of laminates & $\varepsilon_{x}^{T}$ & $\varepsilon_{y}^{T}$ & $\gamma_{x y}^{T}$ & $\kappa_{x}^{T}$ & $\kappa_{y}^{T}$ & $\kappa_{x y}^{T}$ \\
\hline 16-ply laminates & $-6.54 \times 10^{-4}$ & $-6.54 \times 10^{-4}$ & 0 & 0 & 0 & 0 \\
17-ply laminates & $-6.90 \times 10^{-4}$ & $-6.90 \times 10^{-4}$ & 0 & 0 & 0 & 0 \\
\hline
\end{tabular}

effect of single-material laminates and laminates with different proportions of two materials. Table 3 shows the optimization results of two kinds of single-material laminates. Taking 16-ply laminates and 17-ply laminates as an example, the optimized results of laminates with different proportions of two materials are exhibited in Figure 2.

It can be obtained from Table 3 that the maximum extension-shear-coupled effect of glass fiber composite laminates is greater than that of carbon fiber composite laminates in the case of the same number of plies. In the process of optimization by DE_CMSBHS algorithm, if the minimum proportion limits of the two materials are removed, which means the paving material is free, the final optimal results are totally the same as those of single-glass fiber composite laminates of Table 3. Furthermore, the data in Tables 2 and 3 suggests that the maximum extension-shear-coupled effects of the 14-ply, 17-ply, and 20-ply hybrid laminates are greater than those of single-carbon fiber composite laminates, which indicates that the introduction of glass fiber composite materials into carbon fiber composite laminates can improve the extension-shear-coupled effect of partial numbers of plies laminates.

The abscissa " $n_{\mathrm{gl}}$ " in Figure 2 represents the number of glass fiber lamina. As can be seen from Figure 2, (1) for hybrid laminates with different proportions of two materials, there is no feasible solution when $n_{\mathrm{gl}}$ takes some values, for which the geometrical factors of those kinds of laminates cannot satisfy the necessary and sufficient condition of (35) and (2) compared with single-carbon material composite laminates, some hybrid laminates can not only significantly reduce costs but also increase the maximum extensionshear-coupled effect.

\section{Verification of Mechanical Properties of Laminates}

5.1. Verification of Hygrothermal Effect. The finite element method is used to verify the distortion caused by the temperature change of the hybrid $\mathbf{A}_{F} \mathbf{B}_{0} \mathbf{D}_{S}$ laminates with immunity to HTSD. In this paper, the hygrothermal effect of laminates in Table 2 is verified by the example of 16-ply and 17-ply laminates and the conclusion of other laminates is the same as that of these laminates.

Based on the finite element software MSC.Patran, the $10 \mathrm{~m} \times 1 \mathrm{~m}$ finite element model is established and 360 shell units are divided. In order to simulate the displacement boundary condition of the composite laminates, the geometric center of the finite element model is fixed, as shown in Figure 3. The typical temperature difference of the hightemperature curing process is $-180^{\circ} \mathrm{C}$ to this finite element model. Then the finite element software MSC.Nastran is used to compute with the linear statics calculation function.

The calculated results of two kinds of hybrid $\mathbf{A}_{F} \mathbf{B}_{0} \mathbf{D}_{S}$ laminates with immunity to HTSD are shown in Table 4. In which, $\varepsilon_{x}^{T}$ and $\varepsilon_{y}^{T}$ are the thermal strain of two main directions, $\gamma_{x y}^{T}$ is the thermal shear strain of the laminates, and $\kappa_{x}^{T}, \kappa_{y}^{T}$, and $\kappa_{x y}^{T}$ are expressed as the surface curvature and distortion of the laminates caused by temperature changes. It can be seen from the table that the shear strain of these laminates are all zero during the high-temperature curing process, which means two kinds of laminates will not cause hygrothermal distortion. The thermal strains of two directions are equal to each other, but this regulation is not suitable for laminates with different numbers of plies. Furthermore, their bending curvature and twist rate are all zero, which indicates that two kinds of laminates will not cause hygrothermal warping distortion.

5.2. Verification of Extension-Shear-Coupled Effect. In order to verify the extension-shear-coupled effect of two kinds of hybrid $\mathbf{A}_{F} \mathbf{B}_{0} \mathbf{D}_{S}$ laminates in Table 2, the finite element method is also used based on the finite element software MSC.Patran and the finite element model of a rectangular plate with $12 \mathrm{~m} \times 1 \mathrm{~m}$ is established. A total of 800 shell units and 891 nodes (six degrees of freedom) are divided, and a multipoint constraint element (RBE2) is used to connect the nodes in the $1.2 \mathrm{~m} \times 1 \mathrm{~m}$ region at both ends of the model. The axial tension $F=1000 \mathrm{~N}$ is applied to the multipoint confinement unit to ensure that the intermediate $9.6 \mathrm{~m} \times 1 \mathrm{~m}$ area bears a uniform load. The geometric center of the finite element model is fixed, as shown in Figure 4. The finite element software MSC.Nastran is used to compute with the linear statics calculation function.

The calculated displacement nephogram of two kinds of hybrid $\mathbf{A}_{F} \mathbf{B}_{0} \mathbf{D}_{S}$ laminates with immunity to HTSD under axial extension force is shown in Figure 5, and the concrete results of distortion are shown in Table 5. It can be seen from the table that under the axial extension force of $1000 \mathrm{~N}$, the two kinds of hybrid $\mathbf{A}_{F} \mathbf{B}_{0} \mathbf{D}_{S}$ laminates not only have axial distortion but also shear distortion and 


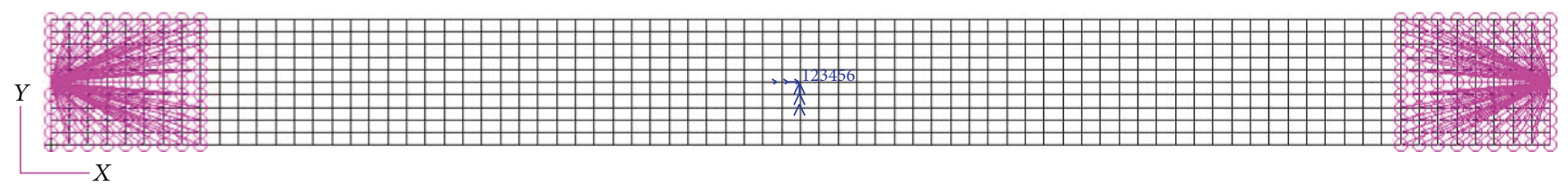

FIGURE 4: Finite element model of the laminates for extension-shear-coupled effect verification.

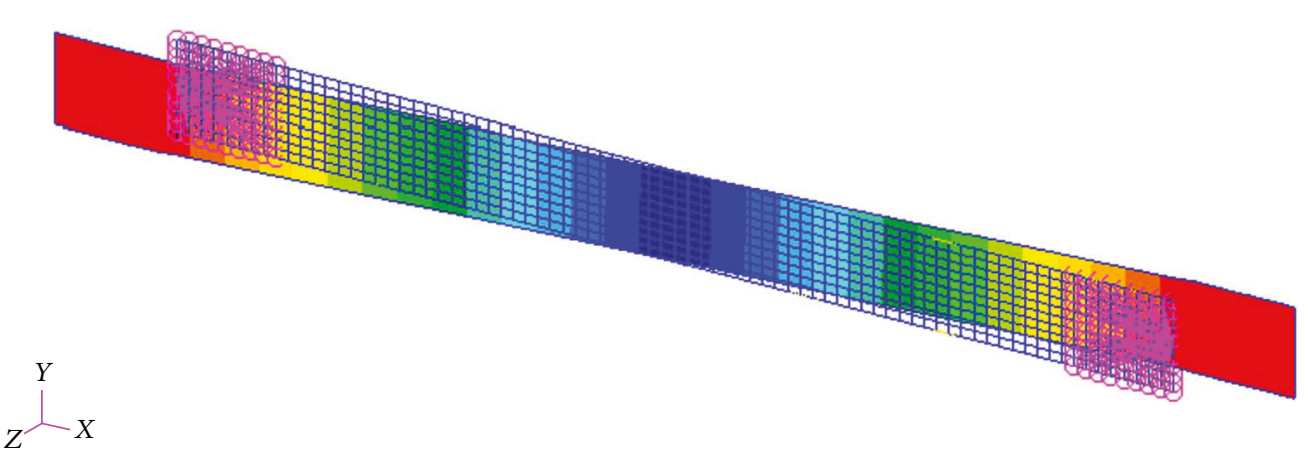

(a) 16-ply laminates

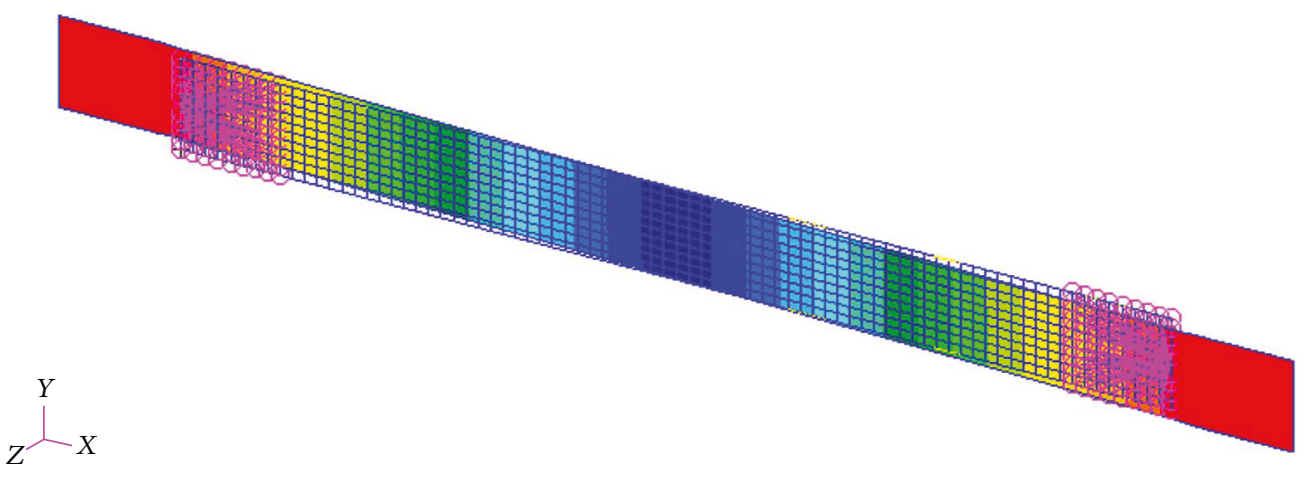

(b) 17-ply laminates
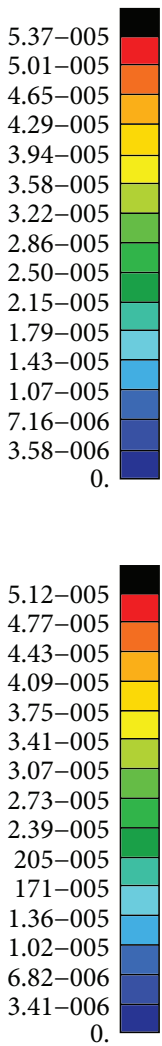

0 .

Figure 5: Deformation of two kinds of hybrid $\mathbf{A}_{F} \mathbf{B}_{0} \mathbf{D}_{S}$ laminates due to extension load.

TABle 5: Simulation results of hybrid $\mathbf{A}_{F} \mathbf{B}_{0} \mathbf{D}_{S}$ laminates on extension-shear distortion.

\begin{tabular}{lcccccc}
\hline Type of laminates & Simulation result $\varepsilon_{x}$ & Theoretical result $\varepsilon_{x}$ & Error of $\varepsilon_{x}$ & Simulation result $\gamma_{x y}$ & Theoretical result $\gamma_{x y}$ & Error of $\gamma_{x y}$ \\
\hline 16-ply laminates & $1.08 \times 10^{-5}$ & $1.10 \times 10^{-5}$ & $1.82 \%$ & $2.23 \times 10^{-6}$ & $2.27 \times 10^{-6}$ \\
17-ply laminates & $1.04 \times 10^{-5}$ & $1.06 \times 10^{-5}$ & $1.89 \%$ & $8.20 \times 10^{-7}$ & $8.26 \times 10^{-7}$ \\
\hline
\end{tabular}

the extension-shear-coupled effect of 16-ply laminates is obviously more than that of 17-ply laminates.

Table 5 also shows the theoretical calculation results of two kinds of hybrid $\mathbf{A}_{F} \mathbf{B}_{0} \mathbf{D}_{S}$ laminates subjected to axial extension distortion, and through comparison, it is found that the results of finite element analysis agree well with the theoretical results. The error is controlled within $2 \%$, and the extension-shear-coupled effect of laminates is validated. The reason for the error is that the finite element simulation is loaded on the unit node and cannot be completely equivalent to the linear loading method.
5.3. Robustness Analysis. Considering that there may be human error and equipment error in the actual process of paving the laminates, which may have a negative effect on the extension-shear-coupled effect, in order to ensure the practicability of the composite laminates, the slight angle deviation should not have an obvious influence on the extension-shear-coupled effect of the hybrid $\mathbf{A}_{F} \mathbf{B}_{0} \mathbf{D}_{S}$ laminates with immunity to HTSD. Now, the extension-shearcoupled effect of laminates is analyzed under the ply angle existing deviation, which is based on the Monte Carlo composite laminates robustness analysis method. 


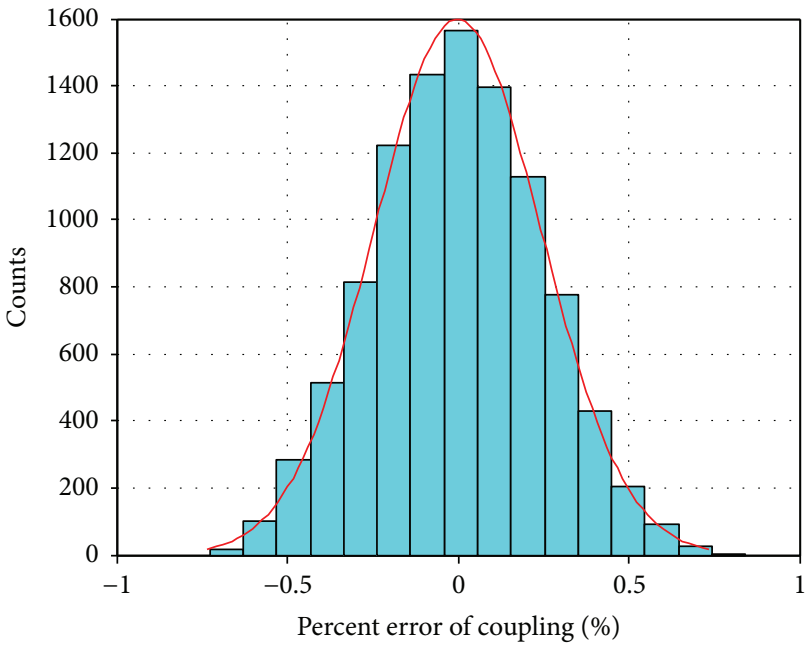

(a) 16-ply laminates

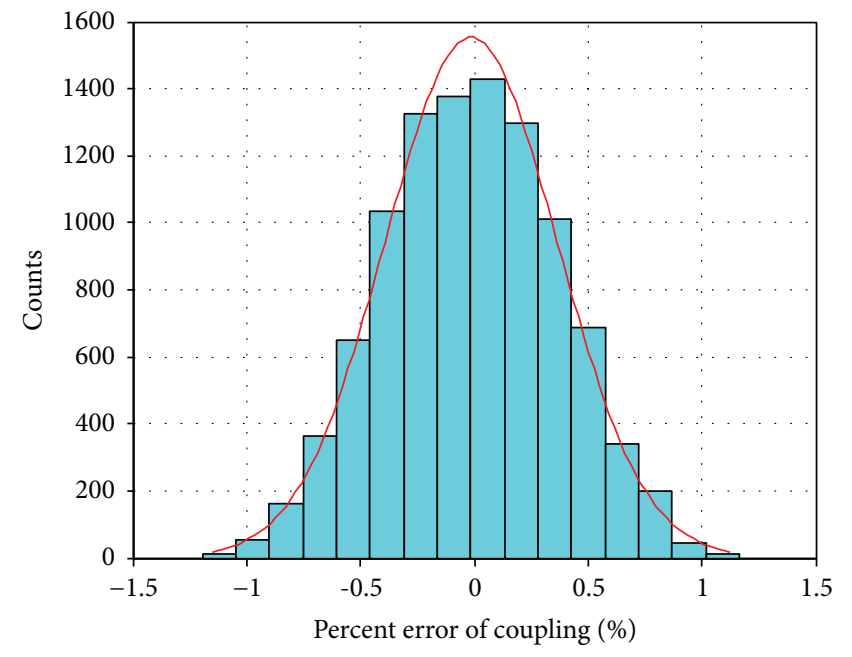

(b) 17-ply laminates

Figure 6: Robustness analysis of hybrid $\mathbf{A}_{F} \mathbf{B}_{0} \mathbf{D}_{S}$ laminates on extension-shear distortion.

Assume that the paving angle of the $k$-ply for the hybrid $\mathbf{A}_{F} \mathbf{B}_{0} \mathbf{D}_{s}$ laminates with immunity to HTSD is $\left(\theta_{k} \pm \Delta \theta_{k}\right)$, in which $\theta_{k}$ is the theoretical paving angle of the $k$-ply and $\Delta \theta_{k}$ is the angle deviation and with the value of $\Delta \theta_{k}=2^{\circ}$ [17]. Figure 6 shows the error distribution of the extensionshear-coupled effect for the 16-ply laminates and the 17-ply laminates in Table 2 under 10,000 random samples. The figure suggests that when there exists a random error in the paving angle of the laminates, the error of extension-shearcoupled effect is in accordance with the normal distribution law and can be controlled within $2 \%$.

\section{Summary}

In this paper, the model of hybrid laminates is established and the expressions of stiffness coefficient, thermal stress, and thermal moment of laminates are derived based on the geometric factor and the necessary and sufficient conditions for hybrid extension-shear-coupled laminates with immunity to HTSD are further derived. The method of combing the improved differential evolution algorithm DE_CMSBHS with the penalty function is used to optimize the extensionshear-coupled effect of the hybrid $\mathbf{A}_{F} \mathbf{B}_{0} \mathbf{D}_{S}$ laminates with immunity to HTSD. The hygrothermal effect, extensionshear-coupled effect, and robustness of laminates are verified. Some conclusions of optimization and simulation can be obtained as follows.

(1) Although the optimized constraints is enhanced, which is mainly due to the introduction of variable parameter paving materials, the feasible solutions of the hybrid $\mathbf{A}_{F} \mathbf{B}_{0} \mathbf{D}_{S}$ laminates with immunity to HTSD can still be obtained by using the DE_CMSBHS algorithm combined with the penalty function. The optimal solution can be obtained according to the different requirements of different proportions of two materials, which achieved the synchronous optimization of paving angle and paving materials.
(2) The maximum extension-shear-coupled effect of the single-glass fiber composite laminates is greater than that of single-carbon fiber composite laminates in the case of the same number of plies, and the introduction of glass fiber composite material into singlecarbon fiber composite laminates can improve the extension-shear-coupled effect of the partial number of plies laminates.

(3) The optimized laminates are able to meet the conditions of no hygrothermal shearing distortion and no hygrothermal warping distortion. When there exists a random error in the paving angle of the laminates, the error of extension-shear-coupled effect is in accordance with the normal distribution law and can be controlled within $2 \%$.

The expressions for parameters of the hybrid laminates can also be applied to laminates with other coupling effects. The DE_CMSBHS algorithm combined with the penalty function can also optimize the different objectives of other types of laminates.

\section{Data Availability}

The data used to support the findings of this study are available from the corresponding author upon request.

\section{Conflicts of Interest}

The authors declare that they have no conflicts of interest.

\section{Acknowledgments}

The authors gratefully acknowledge the support of the National Natural Science Foundation of China (Grant no. 11472003). 


\section{References}

[1] C. B. York, "On extension-shearing coupled laminates," Composite Structures, vol. 120, pp. 472-482, 2015.

[2] J. Li and D. Li, "Extension-shear coupled laminates with immunity to hygro-thermal shearing distortion," Composite Structures, vol. 123, pp. 401-407, 2015.

[3] D. Berry and T. Ashwill, "Design of 9-meter carbon-fiberglass prototype blades: CX-100 and TX-100," SAND2007-0201, Sandia National Laboratories, Warren, RI, USA, 2007.

[4] C. J. Wang and Y. F. Dai, "Application of carbon fiber composite in aerospace," Development \& Innovation of Machinery \& Electrical Products, vol. 2, 2010.

[5] R. M. Jones, Mechanics of Composite Materials, Scripta Book Company, Washington, DC, USA, 1975.

[6] M. Moore and D. Frei, "X-29 forward swept wing aerodynamic overview," in Applied Aerodynamics Conference, Danvers, MA, USA, 1983.

[7] N. J. Krone Jr., "Divergence elimination with advanced composites," in Aircraft Systems and Technology Meeting, Los Angeles, CA, USA, 1974.

[8] V. Tischer and V. Venkayya, "Ply-orientation as a variable in multidisciplinary optimization," in 4th Symposium on Multidisciplinary Analysis and Optimization, Cleveland, OH, USA, 1992.

[9] M. Patil and M. Patil, "Aeroelastic tailoring of composite box beams," in 35th Aerospace Sciences Meeting and Exhibit, Reno, NV, USA, 1997.

[10] S. Guo, "Aeroelastic optimization of an aerobatic aircraft wing structure," Aerospace Science and Technology, vol. 11, no. 5, pp. 396-404, 2007.

[11] S. Adali and V. E. Verijenko, "Optimum stacking sequence design of symmetric hybrid laminates undergoing free vibrations," Composite Structures, vol. 54, no. 2-3, pp. 131-138, 2001.

[12] Y. Zhu, H. Wang, and J. Zhang, "Spacecraft multiple-impulse trajectory optimization using differential evolution algorithm with combined mutation strategies and boundary-handling schemes," Mathematical Problems in Engineering, vol. 2015, Article ID 949480, 13 pages, 2015.

[13] J. A. Snyman, N. Stander, and W. J. Roux, "A dynamic penalty function method for the solution of structural optimization problems," Applied Mathematical Modelling, vol. 18, no. 8, pp. 453-460, 1994.

[14] G. Di Pillo and L. Grippo, “An exact penalty function method with global convergence properties for nonlinear programming problems," Mathematical Programming, vol. 36, no. 1, pp. 1-18, 1986.

[15] C. York, "Unified approach to the characterization of coupled composite laminates: hygro-thermally curvature-stable configurations," International Journal of Structural Integrity, vol. 2, no. 4, pp. 406-436, 2011.

[16] C. B. York, "Tapered hygro-thermally curvature-stable laminates with non-standard ply orientations," Composites Part A: Applied Science and Manufacturing, vol. 44, pp. 140-148, 2013.

[17] R. J. Cross, R. A. Haynes, and E. A. Armanios, "Families of hygrothermally stable asymmetric laminated composites," Journal of Composite Materials, vol. 42, no. 7, pp. 697-716, 2008. 


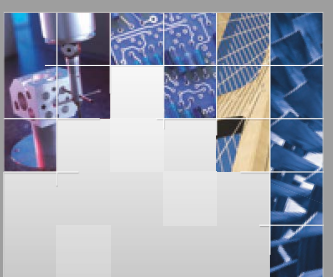

\section{Enfincering}
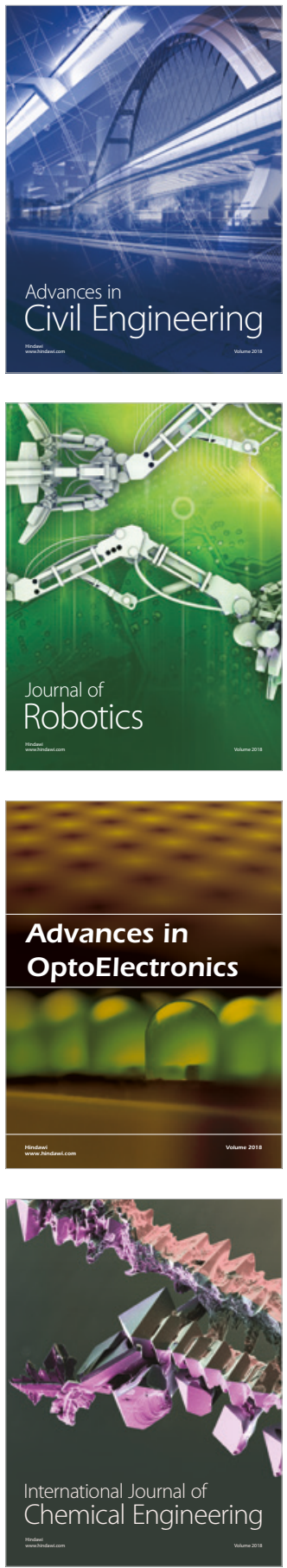

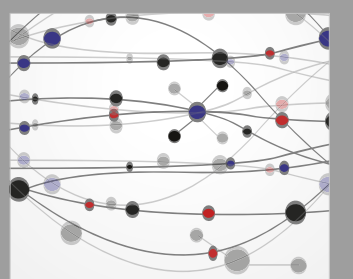

\section{Rotating \\ Machinery}

The Scientific World Journal

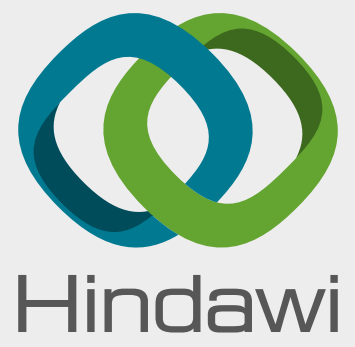

Submit your manuscripts at

www.hindawi.com
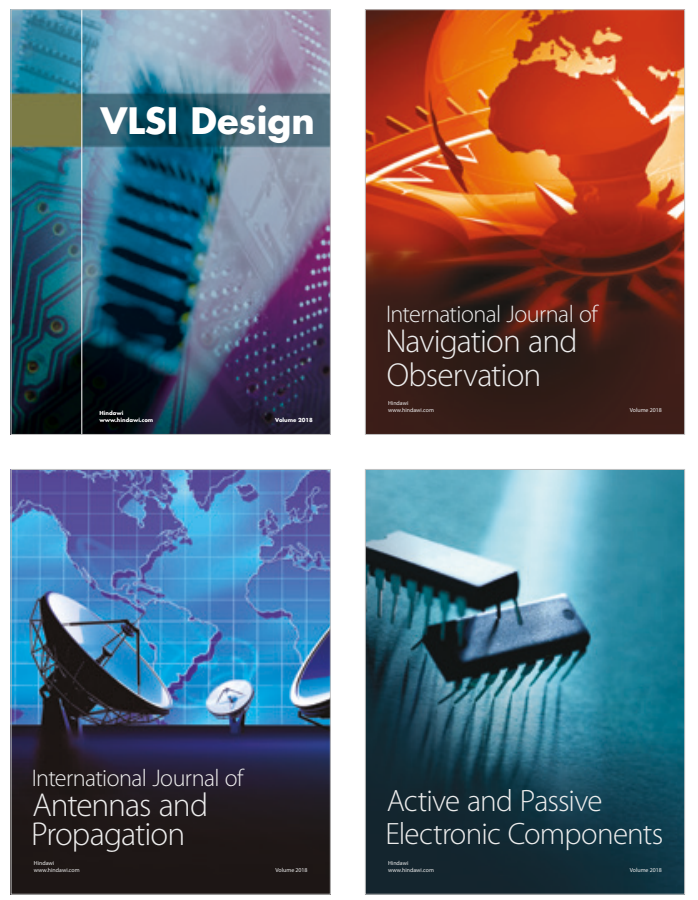
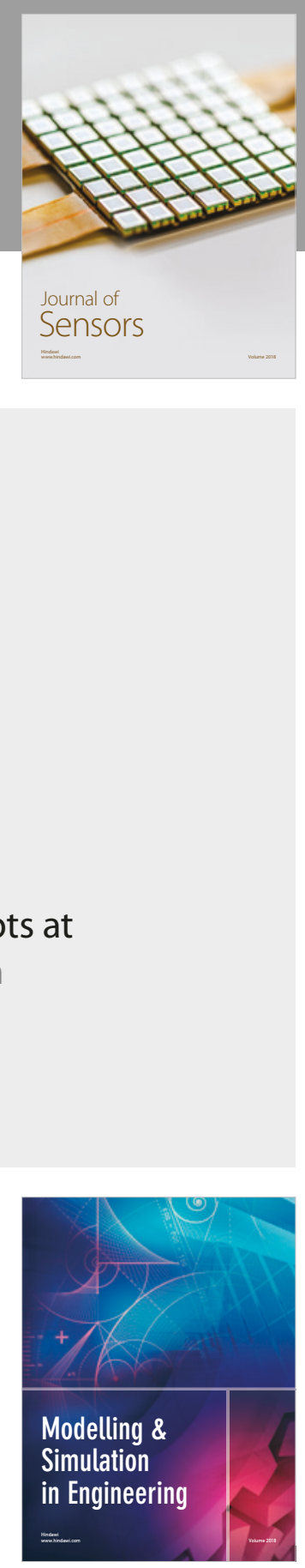

\section{Advances \\ Multimedia}
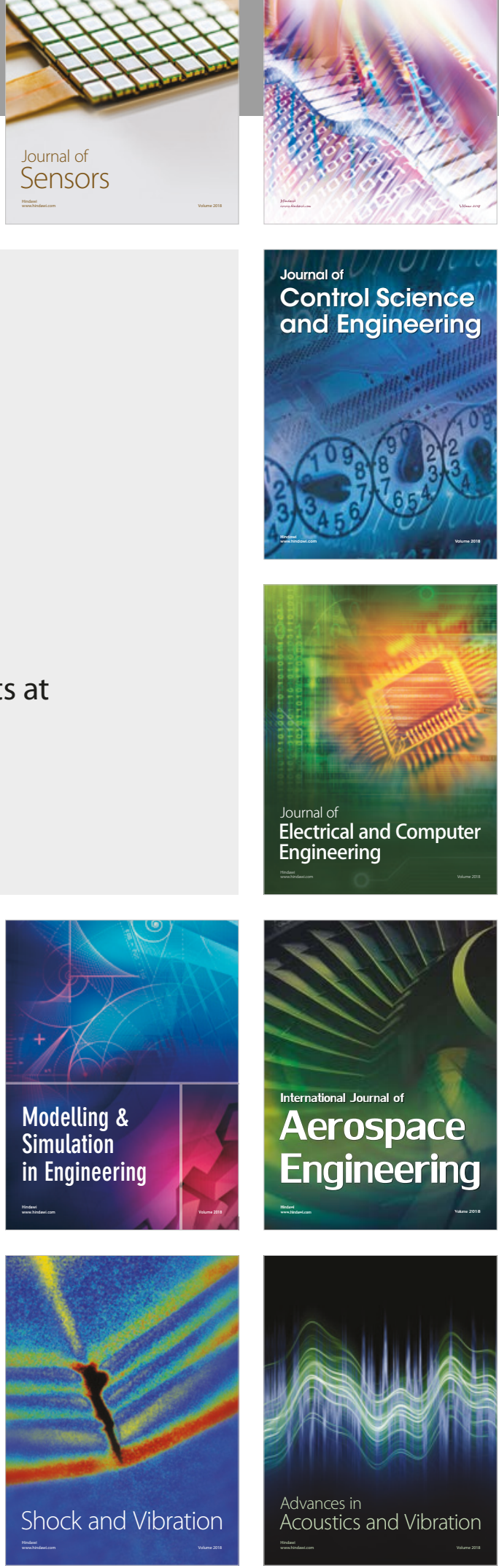